\title{
Random walks on semaphore codes and delay de Bruijn semigroups
}

\author{
John Rhodes \\ Department of Mathematics, University of California, Berkeley, \\ CA 94720, U.S.A. \\ email: rhodes@math.berkeley.edu,BlvdBastille@aol.com \\ Anne Schilling \\ Department of Mathematics, University of California, Davis, \\ One Shields Ave., Davis, CA 95616-8633, U.S.A. \\ email: anne@math.ucdavis.edu \\ Pedro V. Silva \\ Centro de Matemática, Faculdade de Ciências, Universidade do Porto, \\ R. Campo Alegre 687, 4169-007 Porto, Portugal \\ email: pvsilva@fc.up.pt
}

December 30, 2017

\begin{abstract}
We develop a new approach to random walks on de Bruijn graphs over the alphabet $A$ through right congruences on $A^{k}$, defined using the natural right action of $A^{+}$. A major role is played by special right congruences, which correspond to semaphore codes and allow an easier computation of the hitting time. We show how right congruences can be approximated by special right congruences.
\end{abstract}

\section{Introduction}

In graph theory, a $k$-dimensional de Bruijn graph over the alphabet $A$ is a directed graph representing overlaps between sequences of symbols $[9,10]$. The de Bruijn graph has $|A|^{k}$ vertices, given by all words of length $k$ in the alphabet $A$. There is an edge from vertex $a_{1} \ldots a_{k} \in A^{k}$ to vertex $a_{2} \ldots a_{k} a \in A^{k}$ for every $a \in A$. An important question for cryptography and networking is that of de Bruijn sequences. A de Bruijn sequence is a cyclic word of length $|A|^{k}$ such that every possible word of length $k$ over the alphabet $A$ appears once and exactly once (see [16] for a review on de Bruijn sequences). Obviously, a de Bruijn sequence corresponds to a Eulerian path in the de Bruijn graph.

Here we are interested in random walks on the de Bruijn graph $\Gamma$. To an edge $v \stackrel{a}{\longrightarrow} w$ in $\Gamma$ we associate a probability $0 \leq \pi(a) \leq 1$, satisfying $\sum_{a \in A} \pi(a)=1$. This gives rise to the de BruijnBernoulli process (see for example [5, 2]): if we are at vertex $v$ at a given time, then with probability $\pi(a)$ we go to vertex $w$ where $v \stackrel{a}{\longrightarrow} w$ is an edge in $\Gamma$. The transition matrix $\mathcal{T}=\left(\mathcal{T}_{v, w}\right)_{v, w \in A^{k}}$ encodes the transition probabilities, that is, $\mathcal{T}_{v, w}=\pi(a)$ if $v \stackrel{a}{\longrightarrow} w$. Given a random walk, an 
important question is to determine the stationary distribution, which intuitively is the state that is reached after taking many steps in the random walk. Mathematically, the stationary distribution is the vector $I$ such that $I \mathcal{T}=I$. In other words, $I$ is the left eigenvector of $\mathcal{T}$ with eigenvalue one. In the case of the de Bruijn-Bernoulli random walk, the stationary distribution $I \in A^{k}$ is multiplicative [5]

$$
I=\left(\prod_{a \in w} \pi(a)\right)_{w \in A^{k}}
$$

We can reformulate the random walk on the de Bruijn graph in algebraic terms. Namely, let us define the right action of $A$ on $A^{k}$ by

$$
a_{1} \ldots a_{k} \cdot a=a_{2} \ldots a_{k} a
$$

for $a_{1} \ldots a_{k} \in A^{k}$ and $a \in A$. This induces the action of the semigroup $F(|A|, k):=A^{1} \cup A^{2} \cup \cdots \cup A^{k}=$ $A^{\leq k}$ of all words in $A$ of length $1,2, \ldots, k$ with the multiplication - being concatenation and taking the last $k$ letters if the length is bigger than $k$. For example, if $A=\{a, b\}$ and $k=3$, we have $a b \cdot b a=b b a$ in $F(2,3)$. In this formulation, it is clear that the walk in $j$ steps given by $a_{1} \cdots a_{j}$ acts as a constant map (i.e., is independent of the initial vertex) if and only if $j=k$. We call such elements resets.

Random walks on de Bruijn graphs are a "classical" subject. However, in applications it is right congruences $^{1}[1,14,15,19]$ on $A^{k}$ (denoted by $\mathrm{RC}\left(A^{k}\right)$ ) under the faithful action of $F(|A|, k)$ and the associated random walks on their congruence classes that are important. Intuitively, these are the finite semigroups for which any product of $k$ elements act like constant maps on $A^{k}$, but because of the right congruence some products of length less than $k$ might be constant. Right congruences are a standard idea in finite state machines or finite automata theory [18]. In finite state machines, they are used in passing to the unique minimal automata doing the same computation. For example, assume one has a stream of data (e.g. chemical data on waste water being emptied into a river). Assume that there exist a positive integer $k$, so that only the $k$ most recent symbols of data matter. Then there is a function $f: A^{k} \rightarrow D$, where $D$ is the data set. The function could be of the form $f\left(a_{1}, \ldots, a_{k}\right)$ is ok or not ok (that is, $D$ is a two element set) depending on whether this recent $k$ long data meets EPA standards. Then the function $f$ gives an equivalence relation $\sim$ on $A^{k}$ given by $s \sim t$ if and only if $f(s)=f(t)$. In addition, there is a unique maximal refinement of $\sim$ which is a right congruence (that is, the best lower approximation by a right congruence) $R$, namely $s R t$ for $s, t \in A^{k}$ if and only if for all strings $u \in A^{*}$ we have $s . u \sim t$.u or equivalently $f(s u)=f(t u)$. Here . is the multiplication in $F(|A|, k)$. Then $\left(A^{k} / R, F(|A|, k)\right)$ can compute the function $f$ since $f$ factors through the $R$ classes (take $u$ to be 1 ). See [18] for more details.

Consider the right congruence in $\operatorname{RC}\left(A^{3}\right)$ with $A=\{a, b\}$ defined by the congruence classes

$$
\{a a a, b a a, a b a\},\{b b a\},\{a a b, b a b\},\{a b b\},\{b b b\} .
$$

It is not hard to check that if $w, v \in A^{3}$ are in the same congruence class, then $w \cdot z$ and $v \cdot z$ for $z \in F(2,3)$ are also in the same congruence class, proving that (1.1) is indeed in $\operatorname{RC}\left(A^{3}\right)$. The

\footnotetext{
${ }^{1}$ An equivalence relation is a right congruence if it preserves the right action of a semigroup. See Definition 2.2 for more details.
} 


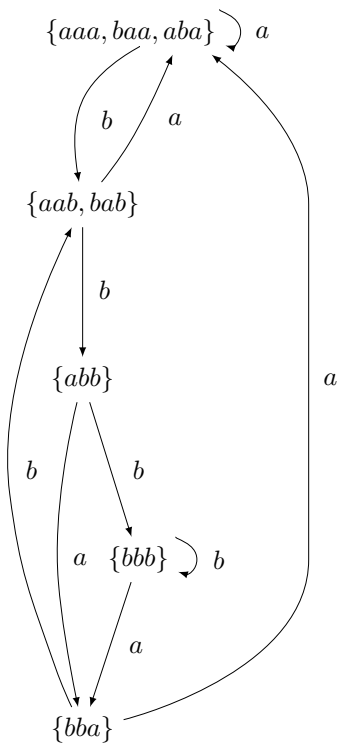

Figure 1.1: The transition graph for the congruence of Equation (1.1).

transition graph is given in Figure 1.1 and the transition matrix of the associated random walk is

$$
\mathcal{T}=\left(\begin{array}{ccccc}
\pi(a) & 0 & \pi(b) & 0 & 0 \\
\pi(a) & 0 & \pi(b) & 0 & 0 \\
\pi(a) & 0 & 0 & \pi(b) & 0 \\
0 & \pi(a) & 0 & 0 & \pi(b) \\
0 & \pi(a) & 0 & 0 & \pi(b)
\end{array}\right)
$$

By lumping $[12,13]$, we can obtain the stationary distribution for $\mathcal{T}$ from the stationary distribution of the de Bruijn-Bernoulli stationary distribution by adding the product distributions for each member of a congruence class. In our example

$$
\begin{aligned}
I & =\left(\pi(a)^{3}+2 \pi(a)^{2} \pi(b), \pi(a) \pi(b)^{2}, \pi(a)^{2} \pi(b)+\pi(a) \pi(b)^{2}, \pi(a) \pi(b)^{2}, \pi(b)^{3}\right) \\
& =\left(\pi(a)^{2}+\pi(a)^{2} \pi(b), \pi(a) \pi(b)^{2}, \pi(a) \pi(b), \pi(a) \pi(b)^{2}, \pi(b)^{3}\right),
\end{aligned}
$$

where for the second line we used that $\pi(a)+\pi(b)=1$.

Recall that all elements in $F(|A|, k)$ of length $k$ are constant maps. We are interested in the probability that an element of length $1 \leq \ell<k$ is a constant map when $F(|A|, k)$ acts on right congruences. This is intuitively related to the hitting time (or waiting time) to constant map. As we will show in Section 6, there is a lattice structure imposed on the set of right congruences with partial order being inclusion. It turns out that we can approximate right congruences by special right congruences as introduced in Section 7 using certain meets and joins in this lattice. Special right congruences in turn are associated to semaphore codes as defined in Section 4, on which it is easy to compute the hitting time (see Section 8). The hitting time of the approximation (given by a semaphore code) and the right congruence turn out to be the same, and the approximation is finer than the right congruence. The stationary distributions of the two are simply related by "lumping". 
Let us now turn our attention to semaphore codes. For a fixed alphabet $A$, which we assume to be a finite non-empty set, denote by $A^{+}$the set of all strings $a_{1} \ldots a_{\ell}$ of length $\ell \geq 1$ over $A$ with multiplication given by concatenation. Thus $\left(A^{+}, A\right)$ is the free semigroup with generators $A$ (since every semigroup $(S, \cdot)$ generated by a subset $A \subseteq S$ is a surmorphism of $\left(A^{+}, A\right)$ by mapping $\left.a_{1} \ldots a_{\ell} \rightarrow a_{1} \cdot a_{2} \cdot \ldots \cdot a_{\ell} \in S\right)$. Furthermore, let $A^{*}=A^{+} \cup\{1\}$, so that $A^{*}$ is $A^{+}$with the identity added; it is the free monoid generated by $A$. The semigroup $A^{+}$has three orders: "is a suffix", "is a prefix", and "is a factor". In particular, for $u, v \in A^{+}$

$$
\begin{aligned}
& u \text { is a suffix of } v \quad \Longleftrightarrow \exists w \in A^{*} \text { such that } w u=v, \\
& u \text { is a prefix of } v \quad \Longleftrightarrow \exists w \in A^{*} \text { such that } u w=v, \\
& u \text { is a factor of } v \Longleftrightarrow \exists w_{1}, w_{2} \in A^{*} \text { such that } w_{1} u w_{2}=v .
\end{aligned}
$$

A suffix code $C$ of $A^{+}$(or over $A$ ) is a subset $C \subseteq A^{+}$so that all elements in $C$ are pairwise incomparable in the suffix order [6].

A semaphore code [6] is a suffix code $S$ over $A$ for which there is a right action in the following sense:

If $u \in S \subseteq A^{+}$and $a \in A$, then $u a$ has a suffix in $S$ (and hence a unique suffix of $u a$ ).

The right action $u . a$ is the suffix of $u a$ that is in $S$.

(The dual concept of prefix codes and left actions is often used in the literature, see for example [6]). For example, $S=\left\{b a^{j} \mid j \geq 0\right\}=: b a^{*}$ is an infinite semaphore code with right action

$$
b a^{j} \cdot a=b a^{j+1} \quad \text { and } \quad b a^{j} \cdot b=b .
$$

In practice, to check whether a suffix code is a semaphore code one merely needs to check the first line of (1.2). For example, $C=\{a, b b\}$ is a suffix code, but $a . b$ has no suffix in $C$, so that $C$ is not a semaphore code.

Semaphore codes over $A$ are inherently related to ideals of $A^{+}$. A subset $I \subseteq A^{+}$is an ideal if $u I v \subseteq I$ for all $u, v \in A^{*}$. Similarly, $L \subseteq A^{+}$is a left ideal if $u L \subseteq L$ for all $u \in A^{*}$. In this setting, suffix codes over $A$ are precisely the suffix minimal elements of a left ideal $L$.

Now given an ideal $I \subseteq A^{+}$we construct a semaphore code as follows. Given $u=a_{j} \ldots a_{2} a_{1} \in A^{+}$, check whether $u$ is in $I$. If $u \notin I$, ignore $u$. If $u \in I$, we find the (necessarily unique) index $1 \leq i \leq j$ such that $a_{i-1} \ldots a_{1} \notin I$, but $a_{i} \ldots a_{1} \in I$. Then $a_{i} \ldots a_{1}$ is a code word and the set of all such words forms the semaphore code $S=: I \beta_{\ell}$, as can be readily verified. It is easy to show that

$$
I \longleftrightarrow I \beta_{\ell}
$$

is a bijection between ideals $I \subseteq A^{+}$and semaphore codes over $A$, see Proposition 4.3. Hence semaphore codes are precisely the suffix minimal elements of an ideal $I \subseteq A^{+}$. Since ideals are ubiquitous in mathematics, so are semaphore codes!

As mentioned earlier, the set of right congruences $\mathrm{RC}\left(A^{k}\right)$ is a finite lattice under the inclusion order on the congruence classes, where the meet is given by intersection. We prove that $\operatorname{RC}\left(A^{k}\right)$ is semimodular, but not modular in general, and thus satisfies the Jordan-Dedekind condition that all maximal chains are of the same length. Also for $|A| \geq 2$ and $k \geq 2, \operatorname{RC}\left(A^{k}\right)$ is not generated by its atoms. See Section 6 for more details. 
Denote by $\operatorname{Sem}\left(A^{k}\right)$ the set of semaphore codes coming from ideals $I \supseteq A^{k}$. This means that all codewords of $\operatorname{Sem}\left(A^{k}\right)$ have length less than or equal to $k$ (so the code is finite) and every member of $A^{k}$ has a suffix in the code. Starting with a semaphore code $S$ and restricting the codewords of $S$ to those of length $\leq k$, might not yield a finite semaphore code. But it is always possible to add codewords of length $k$ to this length restricted semaphore code to obtain $S_{k} \in \operatorname{Sem}\left(A^{k}\right)$. This process of adding codewords of length $k$ which have no suffix in the restricted words is unique. For example, we have seen that $S=b a^{*}$ is a semaphore code. If we take $k=3$, we obtain $\left\{b, b a, b a^{2}\right\}$. However, aaa has no suffix in this set, so it needs to be added to obtain the restricted semaphore code $S_{3}=\{b, b a, b a a, a a a\}$. In [22] we show that if $S$ is a semaphore code, then the finite semaphore code $S_{k}$ converges to $S$ in some precise sense.

Now each semaphore code $S \in \operatorname{Sem}\left(A^{k}\right)$ gives a right congruence $\rho \in \mathrm{RC}\left(A^{k}\right)$ as follows:

For two strings $u, v \in A^{k}$, we say $u \sim_{S} v$ if $u$ and $v$ have a common suffix in $S$.

It is not too hard to verify that $\sim_{S}$ defines a right congruence on $A^{k}$. For example, for $A=\{a, b\}$

$$
S=\{a a, a b, a b a, b b a, a b b, b b b\} \in \operatorname{Sem}_{3}(A)
$$

yields the right congruence in $\mathrm{RC}\left(A^{3}\right)$

$$
\{a a a, b a a\},\{a a b, b a b\},\{a b a\},\{b b a\},\{a b b\},\{b b b\} .
$$

We denote all elements of $\operatorname{RC}\left(A^{k}\right)$ that arise from semaphore codes in $\operatorname{Sem}\left(A^{k}\right)$ by $\operatorname{SRC}\left(A^{k}\right)$, the special right congruences of $\mathrm{RC}\left(A^{k}\right)$. We prove in Section 7 that $\operatorname{SRC}\left(A^{k}\right)$ is a full (meaning that top and bottom agree) sublattice of $\mathrm{RC}\left(A^{k}\right)$, so that each element $\rho \in \mathrm{RC}\left(A^{k}\right)$ has a unique largest lower (finer) approximation denoted by $\rho$, namely $\rho$ is the join of all elements in $\operatorname{SRC}\left(A^{k}\right)$ contained in $\rho$. We will also prove in Section 7 , and the reader can verify this, that the right congruence in (1.1) is not a special right congruence, but the special right congruence in (1.4) is the unique lower approximation.

As for the de Bruijn graphs, we have random walks on semaphore codes since there is a right action of a semigroup on semaphore codes. If $S$ is a semaphore code over the alphabet $A$ and $\pi: A \rightarrow[0,1]$ is any probability distribution on $A$, namely $\sum_{a \in A} \pi(a)=1$, then [6, Proposition 3.5.1]

$$
\sum_{s \in S} \pi(s)=1
$$

where $\pi(s)=\pi\left(a_{1}\right) \cdots \pi\left(a_{\ell}\right)$ if $s=a_{1} \ldots a_{\ell}$. This means in particular that $S$ is a maximal code with respect to inclusion.

We can now construct a random walk with state space given by the code words in $S$ using the right action given in (1.2). Defining the $|S| \times|S|$ monomial matrix $\mathcal{T}(a)$ for each $a \in A$ by $\mathcal{T}(a)_{s, s . a}=1$ and 0 otherwise for all $s \in S$, we obtain the transition matrix as

$$
\mathcal{T}=\sum_{a \in A} \pi(a) \mathcal{T}(a)
$$

We prove in Theorem 8.1 that the stationary distribution $I$ of $\mathcal{T}$ is given by $I=(\pi(s))_{s \in S}$. Furthermore, the probability that a word of length $\ell$ is a reset (or constant map) is

$$
P(\ell)=\sum_{\substack{s \in S \\ \ell(s) \leq \ell}} \pi(s),
$$


see Theorem 8.2. This probability is related to the hitting time to reset. For example, for the semaphore code $S=b a^{*}$, all words $w$ are resets unless $w=a^{\ell}$. The probability that a string of length 3 is a reset is $P(3)=\pi(b)+\pi(b) \pi(a)+\pi(b) \pi(a)^{2}=1-\pi(a)^{3}$. For more details see Section 8 .

We are now able to give a more direct construction of the special right congruence $\underline{\rho}$ for $\rho \in$ $\operatorname{RC}\left(A^{k}\right)$, the best lower approximation of $\rho$ in $\operatorname{SRC}\left(A^{k}\right)$. Define

$$
\operatorname{Res}(\rho)=\left\{w \in A^{+} \mid w \text { is a reset on } A^{k} / \rho\right\} .
$$

Then we prove that $\operatorname{Res}(\rho)$ is an ideal of $A^{k} \subseteq A^{+}$and the special right congruence associated to the semaphore code given by this ideal is $\rho$. An immediate consequence is that $\rho$ and $\rho$ have the same hitting time to reset, but in general different stationary distributions. In general, $\rho$ has more congruence classes than $\rho$, so the stationary distributions cannot be the same. Note that both distributions are determined by lumping from the product distribution of the de Bruijn random walk on $A^{k}$. In applications a metric is placed on all distributions of $\operatorname{RC}\left(A^{k}\right)$. Then the probability distribution $\pi$ on $A$ is chosen such that the distance between $I_{\rho}$ and $I_{\underline{\rho}}$ is minimal. This is called the principle of choosing a "correct" or "good" probability distribution $\pi$ on $A$.

The paper is organized as follows. In Section 2 we provide the algebraic background of the semigroups related to right congruences. The precise definition of resets is given in Section 3 . Semaphore codes are introduced in Section 4. In Sections 5 right congruence and their properties are studied, in particular the lattice structure in Section 6. Special right congruences are the subject of Section 7. Random walks on semaphore codes are studied in Section 8. Note that the semaphore codes introduced in Section 4 can be infinite. The analysis in terms of random walks in Section 8 is valid for both finite and infinite semaphore codes. In all other sections we restrict to finite lengths words and codes.

\section{Acknowledgements}

We would like to thank Arvind Ayyer, Benjamin Steinberg and Nicolas M. Thiéry for discussions.

The first author thanks the Simons Foundation-Collaboration Grants for Mathematicians for travel grant \#313548. The second author was partially supported by NSF grants OCI-1147247 and DMS-1500050. The third author was partially supported by CMUP (UID/MAT/00144/2013), which is funded by FCT (Portugal) with national (MEC) and European structural funds through the programs FEDER, under the partnership agreement PT2020.

\section{Algebraic foundations}

\section{$2.1 \quad$ Elliptic maps on rooted trees}

Elliptic maps on finite trees were considered by Rhodes and Silva [17, 20]. A tree is a connected graph that does not contain a closed walk in which all vertices are distinct. A leaf of a tree is a vertex of degree 1, that is, a vertex that connects to exactly one edge. A rooted tree is a tree in which a particular node is designated as the root. In this case, if a vertex $u$ is on the path from the root to another vertex $v$, we say that $u$ is an ancestor of $v$, or equivalently, that $v$ is a descendant of $u$. If $u$ and $v$ are adjacent, we say that $u$ is the parent of $v$, which is the child of $u$. 


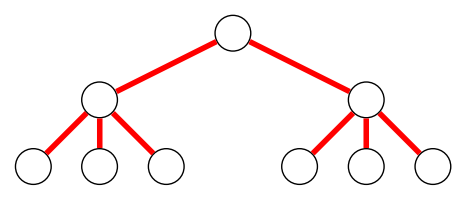

Figure 2.1: Rooted tree $T(2,3)$.
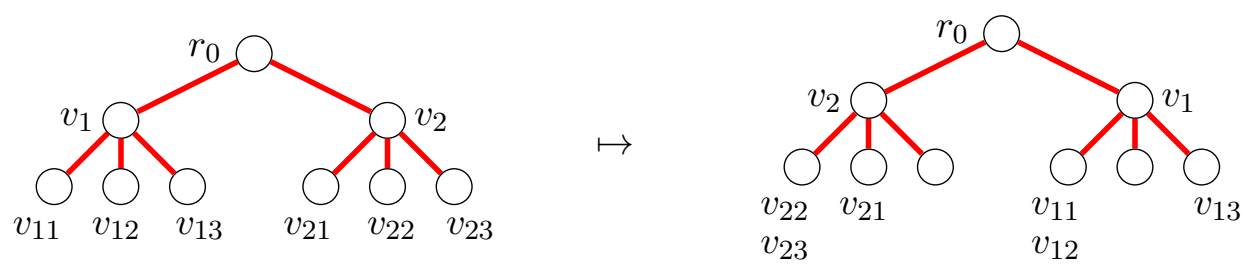

Figure 2.2: Elliptic map $\varphi: \operatorname{Vert}(T) \rightarrow \operatorname{Vert}(T)$ on $T:=T(2,3)$ which maps $r_{0} \mapsto r_{0}, v_{1} \mapsto v_{2}$, $v_{2} \mapsto v_{1}, v_{11} \mapsto v_{21}, v_{12} \mapsto v_{21}, v_{13} \mapsto v_{23}, v_{21} \mapsto v_{12}, v_{22} \mapsto v_{11}, v_{23} \mapsto v_{11}$.

Given a rooted tree $T$, we denote by $\operatorname{Vert}(T)$ the set of vertices of $T$. The distance between two vertices is the minimum number of edges in a path between them. An elliptic map on $T$ is a mapping $\operatorname{Vert}(T) \rightarrow \operatorname{Vert}(T)$ preserving adjacency and distance to the root. Equivalently, an elliptic map on $T$ is a contraction (decreases distances between vertices) while preserving distance to the root, or a mapping fixing the root and preserving parenthood. We shall write functions on the right since we will deal with right actions and compositions. Elliptic maps on a fixed rooted tree form a monoid under composition.

Let $T:=T\left(n_{0}, \ldots, n_{N}\right)$ be a uniformly branching rooted tree, where all leaves are at distance $N+1$ from the root $r_{0}$ and each vertex at distance (or level) $k$ from the root has $n_{k}$ children for $k=0, \ldots, N$. An example of a uniformly branching rooted tree is given in Figure 2.1. An example of an elliptic map on this tree is given in Figure 2.2.

There is another way to represent an elliptic map $\varphi$ using component actions. Namely, a given vertex $v \in \operatorname{Vert}(T)$ at level $k$ is completely specified by the unique path $r_{0} \rightarrow w_{1} \rightarrow \cdots \rightarrow w_{k}=v$ from the root. Since elliptic maps preserve parenthood, the image of this path under the elliptic map $r_{0} \rightarrow\left(w_{1}\right) \varphi \rightarrow \cdots \rightarrow\left(w_{k}\right) \varphi=(v) \varphi$ is again a path, this time from $r_{0}$ to $(v) \varphi$. Hence $\varphi$ can be defined recursively: given the map from path $r_{0} \rightarrow w_{1} \rightarrow \cdots \rightarrow w_{k-1}$ to $r_{0} \rightarrow\left(w_{1}\right) \varphi \rightarrow \cdots \rightarrow\left(w_{k-1}\right) \varphi$, we can define a map $s_{w}$ from the children of $w:=w_{k-1}$ to the children of $\left(w_{k-1}\right) \varphi$. The map $s_{w}$ is called the component action at vertex $w$. Graphically, we place $s_{w}$ on the vertex $w$ for every vertex $w$ that is not a leaf. See Figure 2.4. The elliptic map of Figure 2.2 is written using component actions in Figure 2.3.

As mentioned before, the product of elliptic maps is composition, which is another elliptic map. We can formulate this in terms of the component actions. Let $\varphi$ and $\psi$ be elliptic maps on the same rooted tree $T$ with component action $s_{v}$ and $t_{v}$ at vertex $v \in \operatorname{Vert}(T)$ that is not a leaf, respectively. Then the component action of $\varphi \circ \psi$ at vertex $v$ is $s_{v} t_{(v) s_{w}}$, where $w$ is the parent of $v$. An example is given in Figure 2.5.

Note that a child $v$ of a vertex $w$ can be uniquely specified by the edge $e$ that leads to it. Hence the path $r_{0}=w_{0} \rightarrow w_{1} \rightarrow \cdots \rightarrow w_{k}=v$ from $r_{0}$ to $v$ can alternatively be encoded by a sequence $e_{0} \rightarrow e_{1} \rightarrow \cdots \rightarrow e_{k-1}$ of edges, where $e_{i}$ is the edge from vertex $w_{i}$ to $w_{i+1}$. For us, 


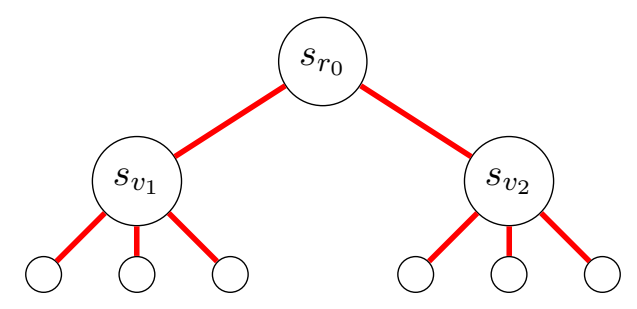

Figure 2.3: Elliptic map of Figure 2.2 written with component actions: $s_{r_{0}}$ is the map $v_{1} \mapsto v_{2}$, $v_{2} \mapsto v_{1}, s_{v_{1}}$ is the map $v_{11} \mapsto v_{21}, v_{12} \mapsto v_{21}, v_{13} \mapsto v_{23}$, and $s_{v_{2}}$ is the map $v_{21} \mapsto v_{12}, v_{22} \mapsto v_{11}$, $v_{23} \mapsto v_{11}$.

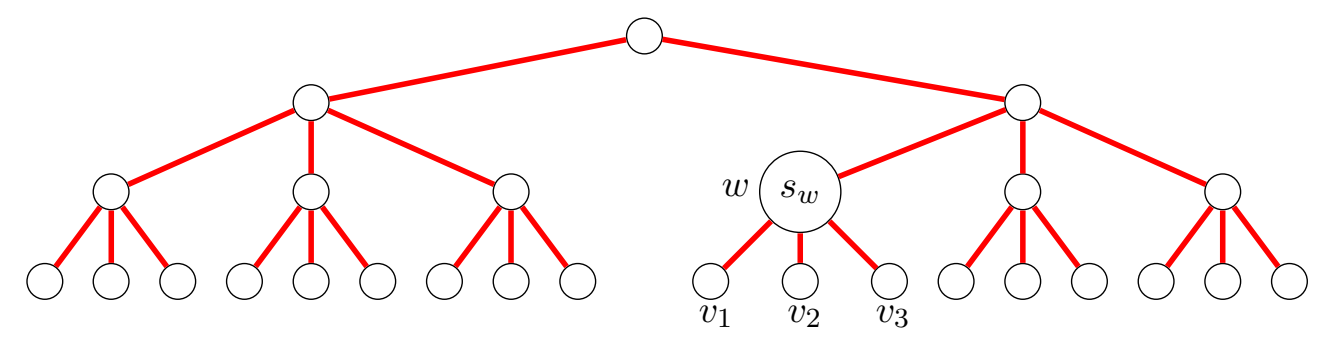

Figure 2.4: Component action at vertex $w$ of an elliptic map on $T(2,3,3)$. The component action $s_{w}$ is a map on the children of $w$, namely on $\left\{v_{1}, v_{2}, v_{3}\right\}$, and maps into the children of the image of $w$ under the elliptic map.

it will be convenient to keep track of the edges by labelling the $n_{\ell}$ edges leaving a given vertex at level $0 \leq \ell \leq N$ bijectively with elements from a set $X_{\ell}$ with $\left|X_{\ell}\right|=n_{\ell}$. The result is a labelled rooted tree. See Figure 2.6 for an example. Note that there are lots of ways to label a rooted tree. Labelling the rooted tree is equivalent to specifying a coordinate system. Once the labelling $L$ of $T$ is fixed, a sequence $e_{0} \rightarrow e_{1} \rightarrow \cdots \rightarrow e_{k-1}$ of edges is determined by an element $\left(x_{0}, x_{1}, \ldots, x_{k-1}\right) \in X_{0} \times X_{1} \times \cdots \times X_{k-1}$.

Given a rooted tree $T\left(n_{0}, \ldots, n_{N}\right)$ with labels in $X=X_{0} \times \cdots \times X_{N}$, elliptic maps can now be expressed using the labels giving rise to the wreath product. The component action at level $k$ is described by a semigroup $S_{k}$ acting faithfully on the right on $X_{k}$, denoted $\left(X_{k}, S_{k}\right)$. Then the wreath product $\left(X_{0}, S_{0}\right) \circ \cdots \circ\left(X_{N}, S_{N}\right)$ is $(X, S)$, where $S$ is the semigroup with component action at level $k$ in $\left(X_{k}, S_{k}\right)$. More precisely, $\Pi=\left(\Pi_{0}, \ldots, \Pi_{N}\right) \in S$ if $\Pi_{0} \in S_{0}, \Pi_{1}: X_{0} \rightarrow S_{1}$, and generally $\Pi_{k}: X_{0} \times \cdots \times X_{k-1} \rightarrow S_{k}$ for $1 \leq k \leq N$, so that for $\left(x_{0}, \ldots, x_{N}\right) \in X$

$$
\left(x_{0}, \ldots, x_{N}\right) \Pi=\left(x_{0} \cdot \Pi_{0}, x_{1} \cdot\left(x_{0}\right) \Pi_{1}, x_{2} \cdot\left(x_{0}, x_{1}\right) \Pi_{2}, \ldots, x_{N} \cdot\left(x_{0}, \ldots, x_{N-1}\right) \Pi_{N}\right) .
$$

The semigroup element $m:=\left(x_{0}, \ldots, x_{k-1}\right) \Pi_{k} \in S_{k}$ is the component action in the vertex (or component) specified by $\left(x_{0}, \ldots, x_{k-1}\right)$.

Remark 2.1 The above arguments show that elliptic maps on uniformly branching trees and wreath products are the same thing (confirming [20, Proposition 3.3]).

Multiplication of wreath products is given by composition of the component action (2.1). Graphically on the level of labelled trees directly, the product $\Pi^{g} \cdot \Pi^{f}$ for $\Pi^{g}, \Pi^{f} \in(X, S)$ translates to the following: 


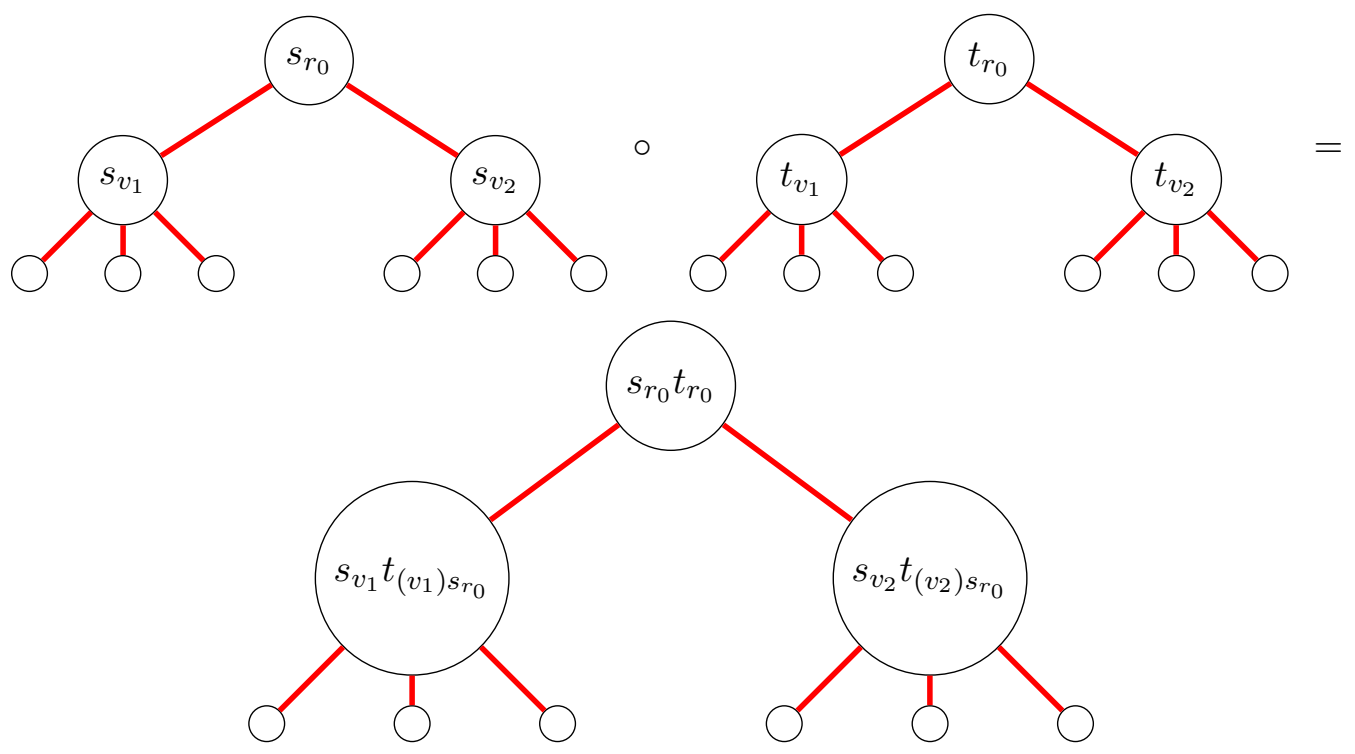

Figure 2.5: Composition or product of two elliptic maps on the rooted tree in Figure 2.1.

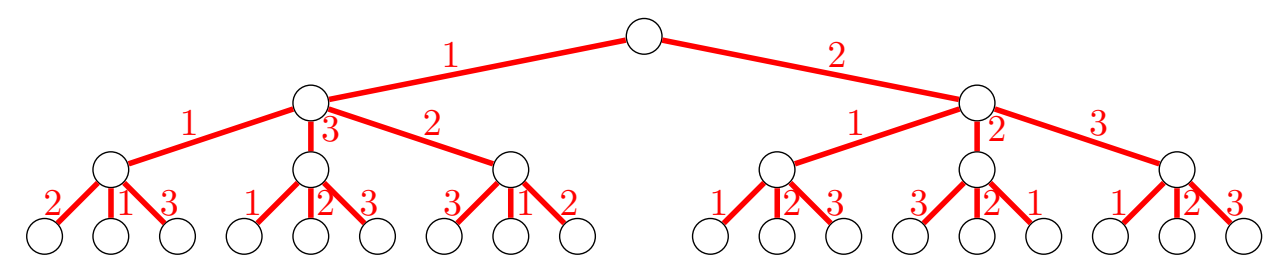

Figure 2.6: Labelled rooted tree $T(2,3,3)$ with labeling sets $X_{0}=\{1,2\}, X_{1}=X_{2}=\{1,2,3\}$.

1. To determine the value of $\Pi^{g} \cdot \Pi^{f}$ at vertex $x=\left(x_{0}, \ldots, x_{k-1}\right)$ in the labelled rooted tree, go to the corresponding vertex in the tree for $\Pi^{g}$, keep track of all values at the vertices on the way and act with the corresponding elements on the vertex vector:

$$
x^{g}=\left(x_{0} \cdot \Pi_{0}^{g}, x_{1} \cdot\left(x_{0}\right) \Pi_{1}^{g}, x_{2} \cdot\left(x_{0}, x_{1}\right) \Pi_{2}^{g}, \ldots, x_{k} \cdot\left(x_{0}, \ldots, x_{k-1}\right) \Pi_{k}^{g}\right) .
$$

2. Then the entry in vertex $\left(x_{0}, \ldots, x_{k-1}\right)$ of $\Pi^{g} \cdot \Pi^{f}$ is $\left(x_{0}, \ldots, x_{k-1}\right) \Pi_{k}^{g}\left(x_{0}^{g}, \ldots, x_{k-1}^{g}\right) \Pi_{k}^{f}$.

One of the main questions is "how restrained can the component action be"? See the first half of [18] and the introduction to [21].

The Prime Decomposition Theorem of Krohn and Rhodes [11] (see also [18] and [21, Chapter 4]) states that every finite semigroup divides an iterated wreath product of its finite simple group divisors and copies of the three element aperiodic monoid $U_{2}$ consisting of two right zeroes and an identity. More precisely, a semigroup $S_{1}$ divides semigroup $S_{2}$, written $S_{1} \mid S_{2}$, if $S_{1}$ is a homomorphic image of a subsemigroup of $S_{2}$. In addition, $U_{2}=\{1, a, b\}$ where $x a=a, x b=b$, and $1 x=x 1=x$ for all $x \in U_{2}$. A finite semigroup is aperiodic if all of its subgroups are trivial. Alternatively, the 


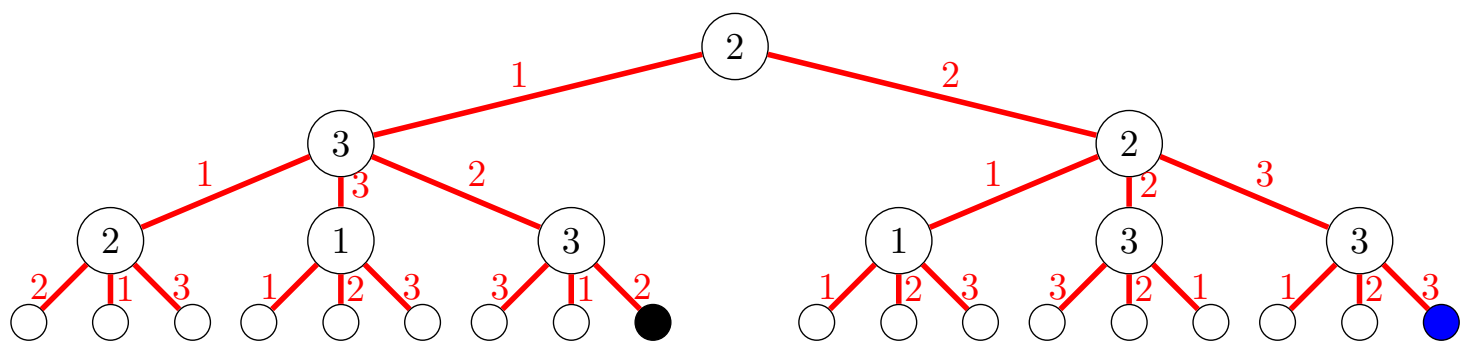

Figure 2.7: Graphical presentation of an elliptic map with $\mathbf{R Z}$ component action using the same labeling as in Figure 2.6. The black leaf has coordinates $(1,2,2)$. Since it passes the constant maps $2,3,3$ on its way, it gets mapped to the leaf with coordinates $(2,3,3)$, denoted by the blue leaf.

Prime Decomposition Theorem says that the basic building blocks of finite semigroups are the finite simple groups and semigroups of constant maps with an adjoined identity.

We say that $I \subseteq S$ is an ideal of the semigroup $S$ if $S I \cup I S \subseteq I$. We write then $I \unlhd S$. The kernel of a semigroup $S$, denoted $\operatorname{ker}(S)$, is the unique minimal nonempty ideal of $S$. If $S$ is a monoid, its group of units is the subgroup formed by all the invertible elements. Both kernel and group of units play a major role in this context.

Let $S_{1}$ and $S_{2}$ be semigroups and let $\varphi$ be a homomorphism of $S_{1}$ into endomorphisms of $S_{2}$. Then the semigroup $S_{1} \times{ }_{\varphi} S_{2}$ is the semidirect product of $S_{1}$ by $S_{2}$ with connecting homomorphism $\varphi$ (see also [21, Section 1.2.2, pg. 23]). More precisely, $S_{1} \times{ }_{\varphi} S_{2}$ has elements in $S_{1} \times S_{2}$ with multiplication given by

$$
\left(s_{1}, s_{2}\right) \cdot\left(s_{1}^{\prime}, s_{2}^{\prime}\right)=\left(s_{1} s_{1}^{\prime}, s_{2}\left(\left(s_{1}^{\prime}\right) \varphi\right) s_{2}^{\prime}\right) .
$$

Notice that wreath products are a special case of semidirect products. In fact, wreath products are "generic" semidirect products. Namely up to pseudovarieties, semidirect products, wreath products, and elliptic products yield the same thing. See [21] for all details.

A semigroup $S$ is called irreducible if for all finite semigroups $S_{1}$ and $S_{2}$ and all connecting homomorphisms $\varphi, S \mid S_{1} \times_{\varphi} S_{2}$ implies $S \mid S_{1}$ or $S \mid S_{2}$. Krohn and Rhodes [11] showed that $S$ is irreducible if and only if either (a) $S$ is a nontrivial simple group; or (b) $S$ is one of the four divisors of $U_{2}$.

A pseudovariety is a collection of finite semigroups closed under taking finite direct products and divisors (that is, subsemigroups and quotients) [21]. The monoid $U_{2}$ is in the pseudovariety $\mathbf{R Z}^{1}$, where $\mathbf{R Z}=[[x y=y]]$ is the pseudovariety of right zeroes, meaning that all elements $x, y$ in $S \in \mathbf{R Z}$ satisfy the identity $x y=y$. In other words, $\mathbf{R Z}$ is the pseudovariety generated by semigroups of constant maps. We denote by $\mathbf{R Z}^{1}$ the pseudovariety generated by semigroups of transformations consisting of constant maps plus the identity mapping. The elements in $\mathbf{R Z}^{1}$ are also called left regular bands, indeed $\mathbf{R} \mathbf{Z}^{1}=\left[\left[x^{2}=x, x y x=y x\right]\right]$ (cf. [21, Proposition 7.3.2]). Random walks on left regular band are an important new topic $[7,8]$. This has recently also been generalized to random walks on $\mathscr{R}$-trivial monoids $[3,4]$.

In light of the Prime Decomposition Theorem, there are three main cases for the component actions in $S_{k}$ of the elliptic maps on $T\left(n_{0}, \ldots, n_{N}\right)$. All of the next three statements have the following form. First note that composition of elliptic maps on a fixed tree with component action in a fixed pseudovariety is closed under composition. Suppose that the component action $S_{k}$ is selected 
to be in the pseudovariety $\mathbf{V}$. Then the pseudovariety generated by elliptic maps with component action in $\mathbf{V}$ (in this case divisors of elliptic maps) is determined and is denoted $\mathbf{P} \mathbf{V}$ (component in $\mathbf{V}$ ). It is the semigroups of $\mathbf{P V}$ (components in $\mathbf{V}$ ) on which we analyze their random walks:

1. $S_{k}$ is in the pseudovariety $\mathbf{R Z}$ with $\mathbf{P V}$ (component in $\mathbf{R Z}$ ) which is delay semigroups (see Section 2.2). In this case the component action consists only of constant maps. If we label the branches from a vertex at level $k$ by $X_{k}=\left\{1,2, \ldots, n_{k}\right\}$, then we can also label the vertices at level $k$ by elements in $X_{k}$. The label $a \in X_{k}$ means the constant map that maps everything to a. An example is given in Figure 2.7.

2. $S_{k}$ is in the pseudovariety $\mathbf{R Z}^{1}$ with $\mathbf{P V}$ (component in $\mathbf{R Z}^{1}$ ) which is aperiodic semigroups (which means semigroups with trivial subgroups). In this case the component action consists of constant maps and the identity; the component monoids are aperiodic. If again the branches at level $k$ are labelled by $X_{k}=\left\{1,2, \ldots, n_{k}\right\}$, then we can label the vertices by elements in $X_{k} \cup\{I\}$, where as before $a \in X_{k}$ denotes the constant map to $a$ and $I$ is the identity.

3. $S_{k}$ is any finite group plus constant maps and $\mathbf{P V}$ (component in any finite group plus constant maps) is all finite semigroups. In this case the vertices at level $k$ are labelled by elements in a finite group $G$ which acts on the right on $X_{k}$ and elements in $X_{k}$ which give the constant maps. This yields a component semigroup with group of units in $G$ and kernel in $\mathbf{R Z}$.

In this paper we will restrict to elliptic maps or wreath products with component actions in RZ, that is constant maps (without identity) to answer the question about resets. Future papers will deal with cases 2 and 3 .

\subsection{Delay pseudovariety}

Let $\mathbf{D}$ be the pseudovariety of semigroups whose idempotents are right zeroes, also called the delay pseudovariety. The pseudovariety $\mathbf{D}$ can be characterized (see [21, pg. 248]) by

$$
\mathbf{D}=\bigcup_{k \geq 1} \mathbf{D}_{k}
$$

where

$$
\mathbf{D}_{k}=\left[\left[x_{0} x_{1} \cdots x_{k}=x_{1} \cdots x_{k}\right]\right],
$$

meaning that any $k+1$ elements $x_{0}, \ldots, x_{k}$ in a semigroup $S \in \mathbf{D}_{k}$ satisfy the identity $x_{0} x_{1} \cdots x_{k}=$ $x_{1} \cdots x_{k}$.

The delay pseudovariety is also equal to $\mathbf{R Z}^{\mathbf{N}}$ defined as

$$
\mathbf{R} \mathbf{Z}^{\mathbf{N}}=\{S \mid S / \operatorname{ker}(S) \text { is nilpotent and } \operatorname{ker}(S) \in \mathbf{R Z}\},
$$

where we recall that $\mathbf{R Z}=[[x y=y]]$. A semigroup $N$ with zero is nilpotent if $N^{k}=\{0\}$ for some $k$, or in other words, $x_{1} \cdots x_{k}=0$ in $N$. Thus, $S \in \mathbf{D}$ if and only if $S$ satisfies the pseudoidentity $x y^{\omega}=y^{\omega}$, where $y^{\omega}$ is the unique idempotent in $\langle y\rangle \leq S$, or more succinctly

$$
\mathbf{D}=\left[\left[x y^{\omega}=y^{\omega}\right]\right]=\mathbf{R} \mathbf{Z}^{\mathbf{N}} .
$$

The pseudovariety $\mathbf{D}$ is also closed under semidirect products. For all details see [21]. 
A semigroup $S$ is a subdirect product of $S_{1}$ and $S_{2}$, denoted $S \ll S_{1} \times S_{2}$, if $S$ is a subsemigroup of $S_{1} \times S_{2}$ mapping onto both $S_{1}$ and $S_{2}$ via the projections [21, pg. 34]. More concretely, $S \ll S_{1} \times S_{2}$ if and only if there exist surmorphisms $\varphi_{i}: S \rightarrow S_{i}$ for $i=1,2$, so that $\varphi_{1}$ and $\varphi_{2}$ separate points, that is, $s, t \in S$ with $s \neq t$ implies that $(s) \varphi_{j} \neq(t) \varphi_{j}$ for some $j \in\{1,2\}$. The right letter mapping congruence on a semigroup $S \in \mathbf{D}$ is defined by $s \sim t$ if $z s=z t$ for all $z \in \operatorname{ker}(S)$, that is, we identify two elements of $S$ if they act the same on the right of $\operatorname{ker}(S)$. Therefore $\sim$ is the kernel of the right Schützenberger representation of $S$ on $\operatorname{ker}(S)$. We denote by RLM: $S \rightarrow S$ the canonical morphism $s \mapsto s / \sim$, and denote its image by $\operatorname{RLM}(S)$. (This definition agrees with the definition given in [21, Section 4.6.2]).

From this it now follows that if $S \in \mathbf{D}=\mathbf{R} \mathbf{Z}^{\mathbf{N}}$, then

$$
S \ll S / \operatorname{ker}(S) \times \operatorname{RLM}(S)
$$

This can be observed by letting $\varphi_{1}: S \rightarrow S / \operatorname{ker}(S)$ be the Rees quotient map, which maps $s \mapsto s$ if $s \notin \operatorname{ker}(S)$ and collapses $\operatorname{ker}(S)$ to a single element. Let $\varphi_{2}: S \rightarrow \operatorname{RLM}(S)$ be the map $s \mapsto s / \sim$. Hence $\varphi_{2}$ is injective on $\operatorname{ker}(S)$, so that $\varphi_{1}$ and $\varphi_{2}$ separate points. In our applications, we only care about $\operatorname{RLM}(S)$. Note that a semigroup $S \in \mathbf{D}$ is nilpotent if and only if $\operatorname{RLM}(S)$ is the trivial semigroup (0).

Observe that for $S, T \in \mathbf{D}$ we have $\operatorname{ker}(S), \operatorname{ker}(T) \in \mathbf{R Z}$ and

$$
\begin{aligned}
& \text { if } \quad S \rightarrow T \text { then } \operatorname{RLM}(S) \rightarrow \operatorname{RLM}(T) \\
& \text { if } S \rightarrow T \text { then } \operatorname{ker}(S) \rightarrow \operatorname{ker}(T) \\
& \operatorname{RLM}(\operatorname{RLM}(S)) \cong \operatorname{RLM}(S) .
\end{aligned}
$$

The proofs are not difficult and all details can be found in [21, Section 4.6.2].

Definition 2.2 An equivalence relation $\tau$ on $\operatorname{ker}(S)$ is called a right congruence if it preserves the right action of $S$ on $\operatorname{ker}(S)$, that is, if $z \tau z^{\prime}$ implies $(z s) \tau\left(z^{\prime} s\right)$ for all $z, z^{\prime} \in \operatorname{ker}(S)$ and $s \in S$. We denote by $\mathrm{RC}(\operatorname{ker}(S), S)$ (or by $\mathrm{RC}(\operatorname{ker}(S)$ ) if $S$ is implicit) the set of all right congruences on $\operatorname{ker}(S)$.

We consider $\mathrm{RC}(\operatorname{ker}(S))$ (partially) ordered by inclusion. Since the intersection of right congruences on $\operatorname{ker}(S)$ is still a right congruence, $(\operatorname{RC}(\operatorname{ker}(S)), \subseteq)$ is a (complete) $\wedge$-semilattice. Thus $(\operatorname{RC}(\operatorname{ker}(S)), \subseteq)$ is indeed a (complete) lattice with the determined join, described by

$$
\vee \Lambda=\bigcap\{\rho \in \operatorname{RC}(\operatorname{ker}(S)) \mid \lambda \subseteq \rho \text { for every } \lambda \in \Lambda\}
$$

for every $\Lambda \subseteq \operatorname{RC}(\operatorname{ker}(S))$.

It is routine to check each $\tau \in \operatorname{RC}(\operatorname{ker}(S), S)$ determines a congruence $\bar{\tau}$ on $(\operatorname{ker}(S), \operatorname{RLM}(S))$ defined by

$$
(s \sim) \bar{\tau}(t \sim) \text { if }(z s) \tau(z t) \text { for every } z \in \operatorname{ker}(S),
$$

where $s \sim$ denotes the equivalence class of $s \in S$ under the right letter mapping congruence $\sim$. Since $S \in \mathbf{D}$, we have $\operatorname{ker}(S) \in \mathbf{R Z}$, and it follows easily that

$$
z \tau z^{\prime} \text { if and only if }(z \sim) \bar{\tau}\left(z^{\prime} \sim\right) \text { holds for all } z, z^{\prime} \in \operatorname{ker}(S) .
$$

Thus right congruences on $\operatorname{ker}(S)$ and right letter mapping images of $S$ are the "same thing". 


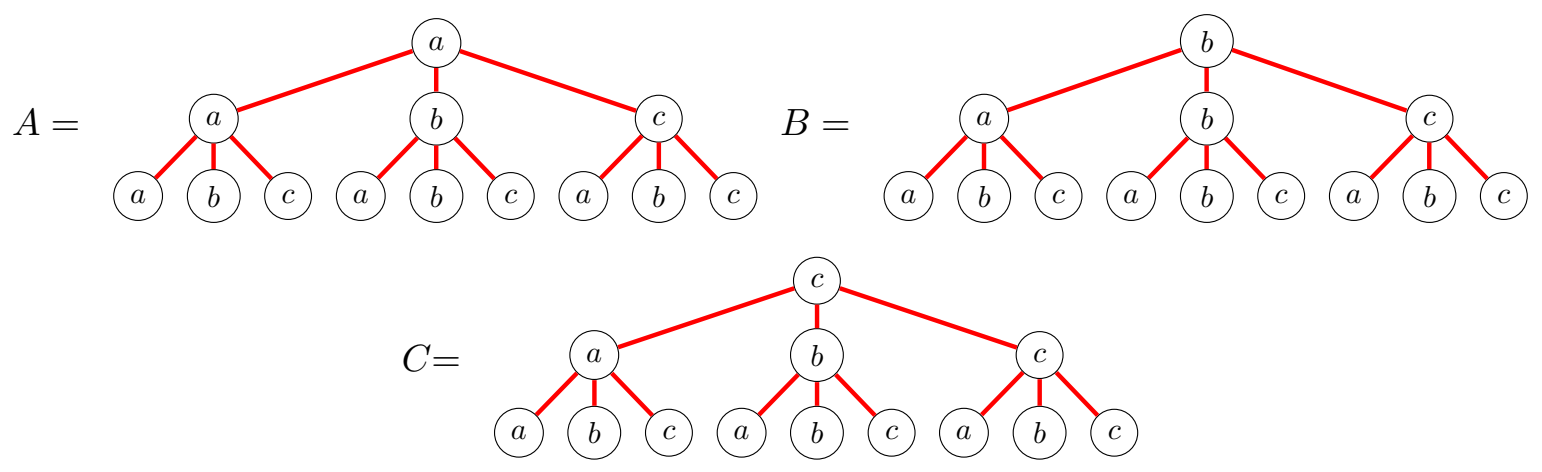

Figure 2.8: Generators for $F(3,3)$ on $T(3,3,3)$ with $A_{3}=\{a, b, c\}$.

\subsection{Right zero component action}

In this section, we specialize the elliptic maps on rooted uniformly branching trees of Section 2.1 to the constant component action. That is, we restrict ourselves to the case that the component action $S_{\ell} \in \mathbf{R Z}=[[x y=y]]$ for all $0 \leq \ell \leq N$.

Let $F(g, k)$ be the semigroup generated by $A_{g}:=\left\{a_{1}, a_{2}, \ldots, a_{g}\right\}$ modulo all relations of the form

$$
a_{i_{0}} a_{i_{1}} \ldots a_{i_{k}}=a_{i_{1}} \ldots a_{i_{k}}
$$

for $i_{0}, \ldots, i_{k} \in\{1, \ldots, g\}$. This semigroup admits a convenient normal form: we can identify $F(g, k)$ with $A^{\leq k} \backslash\{\varepsilon\}$, the set of all nonempty words on $A$ of length at most $k$ (we denote the empty word by $\varepsilon$ ). Note that we may define length of an element of $F(g, k)$ as the length of the respective normal form in $A^{\leq k} \backslash\{\varepsilon\}$.

Given $u \in A^{+}$, let $u \xi_{k}$ denote the suffix of length $k$ of $u$ if $|u| \geq k$ and $u$ otherwise. We define a binary operation $\circ$ on $A^{\leq k} \backslash\{\varepsilon\}$ by

$$
u \circ v=(u v) \xi_{k} .
$$

This binary operation on the normal forms corresponds to the product of $F(g, k)$. For example in $F(2,3)$ with $A_{2}=\{a, b\}$ we have $a b a \cdot a=b a a, a b a \cdot b b b=b b b, b \cdot a=b a$ and so on.

It is immediate that $F(g, k)$ satisfies the identity

$$
x_{0} x_{1} \cdots x_{k}=x_{1} \cdots x_{k} .
$$

Indeed, $F(g, k)$ is the free pro- $\mathbf{D}_{k}$ semigroup over $A$ (see [21, Subsection 3.2.2] for details on free pro-V semigroups, for a pseudovariety $\mathbf{V})$. Since $F(g, k)$ is finite, it follows that $F(g, k) \in \mathbf{D}$. Note that we can identify $\operatorname{ker}(F(g, k))$ with $A^{k}$, the set of all words on $A$ of length $k$.

It can also be interpreted in terms of elliptic maps on $T:=T(\underbrace{g, \ldots, g}_{k})$ as follows. As in Section 2.1, we represent elliptic maps directly on the tree by denoting the component action on the vertices. Define the generators $\varphi_{1}, \ldots, \varphi_{g}$ through trees of depth $k$ with $g$ branches at each level, where in level $1 \leq \ell \leq k$ the vertices are labeled $a_{1}, \ldots, a_{g}$ from left to right. The $i$-th generator has label $a_{i}$ at level 0 . Since the vertices at level $k$ are not labeled, we will omit them for space reasons. An example of the generators for $F(3,3)$ is given in Figure 2.8 . 


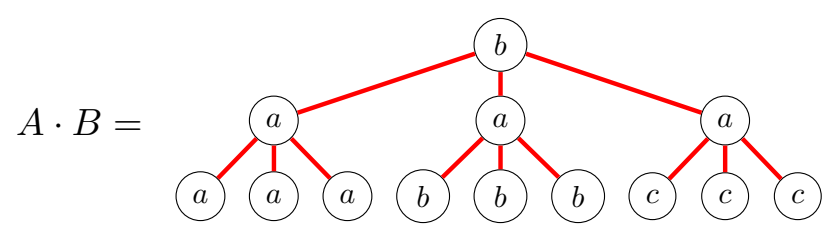

Figure 2.9: Multiplication of elements $A$ and $B$ in $F(3,3)$. Note that the first two levels are constant precisely as specified by $A$ and $B$.

A label $a_{i}$ in a given vertex denotes the constant map to $a_{i}$. If we label the edges under each vertex also $a_{1}, \ldots, a_{g}$ from left to right, then we can multiply generators on the labeled tree as in Section 2.1. See Figure 2.9 for the product of $A$ and $B$ of Figure 2.8. Using the notation $v_{j_{1} \ldots j_{k}}$ to denote the nodes below the root as in Subsection 2.1, we have $v_{j_{1} \ldots j_{k}} \varphi_{i}=v_{i j_{1} \ldots j_{k-1}}$ and so

$$
v_{j_{1} \ldots j_{k}} \varphi_{i_{\ell-1}} \ldots \varphi_{i_{0}}=v_{i_{0} \ldots i_{\ell-1} j_{1} \ldots j_{k-\ell}}
$$

for every $\ell \leq k$. In terms of component actions, this translates into a tree with $a_{i_{0}}$ on level $0, a_{i_{1}}$ on all $g$ vertices of level 1 , and in general $a_{i_{j}}$ on all vertices of level $j$ for $0 \leq j<\ell$. It follows easily from

$$
v_{j_{1} \ldots j_{k}} \varphi_{i_{k-1}} \ldots \varphi_{i_{0}}=v_{i_{0} \ldots i_{k-1}}=v_{j_{1} \ldots j_{k}} \varphi_{i_{k}} \ldots \varphi_{i_{0}}
$$

that $\varphi_{1}, \ldots, \varphi_{g}$ generate a semigroup isomorphic to $F(g, k)$.

This gives a simple proof of Stiffler's Theorem [23] (see also [21, Theorem 4.5.7, pg. 248]).

Theorem 2.3 (Stiffler) The smallest pseudovariety containing the 2-element right zero semigroup that is closed under semidirect product (equivalently wreath or elliptic products) is $\mathbf{D}$.

Proof. As discussed in Section 2.2, D is a pseudovariety that is closed under semidirect product. By the arguments above, the free objects $F(g, k)$ are elliptic products with component action in $\mathbf{R Z}$ and since every member of $\mathbf{D}$ is a suromorphic image of an appropriate free one, the theorem is proved.

In the sequel, we will be interested in the classification of right congruences on $\operatorname{ker}(F(g, k)) \in \mathbf{R Z}$.

\section{$3 \quad k$-reset graphs}

$k$-reset graphs are finite state automata [18] with the additional property that strings of length $k$ are resets or constant maps. The formalism is such that the definitions in the profinite case, when $k$ tends to infinity, is very similar. Let us now discuss the details.

Let $A$ be a finite nonempty alphabet. An $A$-graph is a structure of the form $\Gamma=(Q, E)$, where:

- $Q$ is a finite nonempty set (vertex set);

- $E \subseteq Q \times A \times Q$ (edge set)

A nontrivial path in an $A$-graph $\Gamma=(Q, E)$ is a finite sequence of the form

$$
q_{0} \stackrel{a_{1}}{\longrightarrow} q_{1} \stackrel{a_{2}}{\longrightarrow} \cdots \stackrel{a_{n}}{\longrightarrow} q_{n}
$$


such that $\left(q_{i-1}, a_{i}, q_{i}\right) \in E$ for $i=1, \ldots, n$. Its label is the word $a_{1} a_{2} \cdots a_{n} \in A^{+}=A^{*} \backslash\{\varepsilon\}$, where $A^{*}$ is the set of words in the alphabet $A$ and $\varepsilon$ is the empty word. A trivial path is a formal expression of the form

$$
q \stackrel{\varepsilon}{\longrightarrow} q
$$

An $A$-graph $\Gamma=(Q, E)$ is:

- deterministic if

$$
(p, a, q),\left(p, a, q^{\prime}\right) \in E \Rightarrow q=q^{\prime}
$$

holds for all $p, q, q^{\prime} \in Q$ and $a \in A$;

- complete if

$$
\forall p \in Q \forall a \in A \exists q \in Q:(p, a, q) \in E ;
$$

- strongly connected if, for all $p, q \in Q$, there exists a path $p \stackrel{u}{\longrightarrow} q$ in $\Gamma$ for some $u \in A^{*}$.

If $\Gamma=(Q, E)$ is deterministic and complete, then $E$ induces a function

$$
\begin{aligned}
Q \times A & \rightarrow Q \\
(q, a) & \mapsto q a
\end{aligned}
$$

defined by $(q, a, q a) \in E$. Conversely, every such function defines a deterministic complete $A$-graph. Moreover, we can extend the function $Q \times A \rightarrow Q$ to a function $Q \times A^{*} \rightarrow Q$ as follows: given $q \in Q$ and $u \in A^{*}, q u$ is the unique vertex such that there exists a path

$$
q \stackrel{u}{\longrightarrow} q u
$$

in $\Gamma$. This function is called the transition function of $\Gamma$.

Let $\Gamma=(Q, E)$ and $\Gamma^{\prime}=\left(Q^{\prime}, E^{\prime}\right)$ be $A$-graphs. A morphism $\varphi: \Gamma \rightarrow \Gamma^{\prime}$ is a function $\varphi: Q \rightarrow Q^{\prime}$ such that

$$
(p, a, q) \in E \Rightarrow(p \varphi, a, q \varphi) \in E^{\prime} .
$$

If $\varphi$ is bijective and $\varphi^{-1}$ is also a morphism, we say that $\varphi$ is an isomorphism. In this case we write $\Gamma \cong \Gamma^{\prime}$.

Given $A$-graphs $\Gamma, \Gamma^{\prime}$, we write $\Gamma \leq \Gamma^{\prime}$ if there exists a morphism $\Gamma \rightarrow \Gamma^{\prime}$. This is clearly a reflexive and transitive relation, hence a preorder on the class of all $A$-graphs. Technically, this is not a partial order, but we have the following remark:

Lemma 3.1 Let $A$ be a finite nonempty alphabet and let $\Gamma, \Gamma^{\prime}$ be strongly connected deterministic complete A-graphs such that $\Gamma \leq \Gamma^{\prime} \leq \Gamma$. Then $\Gamma \cong \Gamma^{\prime}$.

Proof. Let $\varphi: \Gamma \rightarrow \Gamma^{\prime}$ and $\varphi^{\prime}: \Gamma^{\prime} \rightarrow \Gamma$ be morphisms. Write $\Gamma=(Q, E)$ and $\Gamma^{\prime}=\left(Q^{\prime}, E^{\prime}\right)$. Fix some $q_{0} \in Q$ and take $q^{\prime} \in Q^{\prime}$. Since $\Gamma^{\prime}$ is strongly connected, there exists some path $q_{0} \varphi \stackrel{u}{\longrightarrow} q^{\prime}$ in $\Gamma^{\prime}$ for some $u \in A^{*}$. Since $\Gamma$ is complete, there exists some path $q_{0} \stackrel{u}{\longrightarrow} q$ in $\Gamma$ for some $q \in Q$. It follows from $\varphi$ being a morphism that there exists a path $q_{0} \varphi \stackrel{u}{\longrightarrow} q \varphi$ in $\Gamma^{\prime}$. Since $\Gamma^{\prime}$ is deterministic, we get $q^{\prime}=q \varphi$, hence $\varphi$ is onto and so $\left|Q^{\prime}\right| \leq|Q|$. By symmetry, we get $\left|Q^{\prime}\right|=|Q|$, thus $\varphi$ is bijective.

It remains to be proved that $\varphi^{-1}$ is a morphism. Assume that $(p \varphi, a, q \varphi) \in E^{\prime}$ for some $p, q \in Q$ and $a \in A$. Since $\Gamma$ is complete, there exists some $(p, a, r) \in E$. Since $\varphi$ is a morphism, we get $(p \varphi, a, r \varphi) \in E^{\prime}$. Now $\Gamma^{\prime}$ being deterministic yields $q \varphi=r \varphi$, and so $q=r$ since $\varphi$ is bijective. Therefore $(p, a, q) \in E$ and so $\varphi^{-1}$ is a morphism as required. 
We say that $u \in A^{*}$ is a reset word for the deterministic and complete $A$-graph $\Gamma=(Q, E)$ if $|Q u|=1$. This is equivalent to say that all paths labeled by $u$ end at the same vertex. Let $\operatorname{Res}(\Gamma)$ denote the set of all reset words for $\Gamma$. For every $k \in \mathbb{N}$, let

$$
\operatorname{Res}_{k}(\Gamma)=\operatorname{Res}(\Gamma) \cap A^{k} .
$$

We say that $\Gamma$ is a $k$-reset graph if $\operatorname{Res}_{k}(\Gamma)=A^{k}$. We denote by $\operatorname{RG}_{k}(A)$ the class of all strongly connected deterministic complete $k$-reset $A$-graphs.

Given $\Gamma \in \mathrm{RG}_{k}(A)$, let $[\Gamma]$ denote the isomorphism class of $\Gamma$. Let

$$
\mathrm{RG}_{k}(A) / \cong=\left\{[\Gamma] \mid \Gamma \in \mathrm{RG}_{k}(A)\right\}
$$

Given $\Gamma, \Gamma^{\prime} \in \mathrm{RG}_{k}(A)$, write

$$
[\Gamma] \leq\left[\Gamma^{\prime}\right] \text { if } \Gamma \leq \Gamma^{\prime}
$$

It is immediate that $\leq$ is a well-defined preorder on $\mathrm{RG}_{k}(A) / \cong$. Moreover, it follows from Lemma 3.1 that:

Corollary 3.2 Let $A$ be a finite nonempty alphabet and let $k \geq 1$. Then $\leq$ is a partial order on $\mathrm{RG}_{k}(A) / \cong$.

\section{Semaphore codes}

A detailed discussion on semaphore codes can be found in [6, Chapter 3.4].

Let $A$ be a finite alphabet. We define three partial orders on $A^{*}$ by

- $u \leq_{p} v$ if $v \in u A^{*}$,

- $u \leq_{s} v$ if $v \in A^{*} u$,

- $u \leq_{f} v$ if $v \in A^{*} u A^{*}$.

We refer to them as the prefix order, the suffix order and the factor order on $A^{*}$.

If $X \subset A^{*}$ is a nonempty antichain with respect to $\leq_{p}$ (respectively $\leq_{s}$, $\leq_{f}$ ), it is said to be a prefix code (respectively suffix code, infix code). Note that our notions differ slightly from the standard notions since we admit $\{\varepsilon\}$ to be a code of all three types!

Given an ideal $I \unlhd A^{*}$, let $I \beta$ denote the subset of elements of $I$ wich are minimal with respect to $\leq_{f}$. Then $I=A^{*}(I \beta) A^{*}$ and $I \beta \subseteq B$ whenever $B \subseteq A^{*}$ satisfies $I=A^{*} B A^{*}$. We say that $I \beta$ is the basis of $I$. Clearly, the correspondences

$$
I \mapsto I \beta, \quad C \mapsto A^{*} C A^{*}
$$

establish mutually inverse bijections between the set of all ideals of $A^{*}$ and the set of all infix codes on $A$.

We say that $L \subseteq A^{*}$ is a left ideal if $L \neq \emptyset$ and $A^{*} L \subseteq L$. We write then $L \unlhd_{\ell} A^{*}$. Given $L \unlhd_{\ell} A^{*}$, let $L \beta_{\ell}$ denote the subset of elements of $L$ wich are minimal with respect to $\leq_{s}$. Then $L=A^{*}\left(L \beta_{\ell}\right)$ and $L \beta \subseteq B$ whenever $B \subseteq A^{*}$ satisfies $L=A^{*} B$. We say that $L \beta_{\ell}$ is the left basis of $L$. Clearly, the correspondences

$$
L \mapsto L \beta_{\ell}, \quad S \mapsto A^{*} S
$$


establish mutually inverse bijections between the set of all left ideals of $A^{*}$ and the set of all suffix codes on $A$.

Similarly, $R \subseteq A^{*}$ is a right ideal if $R \neq \emptyset$ and $R A^{*} \subseteq R$. We write then $R \unlhd_{r} A^{*}$.

We relate now ideals to semaphore codes. The definition we use is actually the left-right dual of the classical definition in [6, Section 3.5], but we shall call them semaphores codes for simplification. We also admit $\emptyset$ and $\{\varepsilon\}$ as (semaphore) codes, but this generalization is compatible with the relevant results from [6].

A semaphore code on the alphabet $A$ is a language of the form

$$
X A^{*} \backslash A^{+} X A^{*}
$$

for some $X \subseteq A^{*}$. If $X \neq \emptyset$, then $X A^{*} \backslash A^{+} X A^{*}$ is a maximal suffix code (with respect to inclusion) by [6, Proposition 3.5.1]. Now [6, Proposition 3.5.4] provides an alternative characterization of semaphore codes:

Lemma 4.1 [6, Proposition 3.5.4] For every $S \subseteq A^{*}$, the following conditions are equivalent:

(i) $S$ is a semaphore code;

(ii) $S$ is a suffix code and $S A \subseteq A^{*} S$.

Let $\operatorname{Sem}(A)$ denote the set of all semaphore codes on the alphabet $A$. We define a partial order $\leq$ on $\operatorname{Sem}(A)$ by $S \leq S^{\prime}$ if $A^{*} S \leq A^{*} S^{\prime}$.

Example 4.2 Let $A=\{a, b\}$ and $X=\{b\}$. Then the semaphore code is infinite

$$
S=X A^{*} \backslash A^{+} X A^{*}=\left\{b, b a, b a^{2}, b a^{3}, \ldots\right\}=b a^{*} .
$$

If on the other hand $A=\{a, b\}$ and $X=\left\{a^{2}, a b, b^{2}\right\}$, then the semaphore code is finite

$$
S=X A^{*} \backslash A^{+} X A^{*}=\left\{a^{2}, a b, b^{2}, a b a, b^{2} a\right\} .
$$

We denote by $\mathcal{I}(A)$ (respectively $\mathcal{L}(A), \mathcal{R}(A)$ ) the set of all ideals (respectively left ideals, right ideals) of $A^{*}$. If we order $\mathcal{I}(A)$ (or $\mathcal{L}(A)$ or $\mathcal{R}(A)$ ) by inclusion, we get a complete (distributive) lattice where meet and join are given by intersection and union. The top element is $A^{*}$ and the bottom element is $\emptyset$. We can now prove the following.

Proposition 4.3 Let $A$ be a finite nonempty alphabet. Then

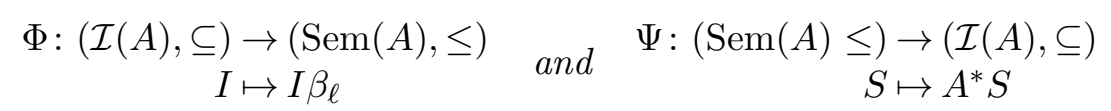

are mutually inverse lattice isomorphisms.

Proof. Let $I \in \mathcal{I}(A)$. Then $I \beta_{\ell}$ is clearly a suffix code. Since $\left(I \beta_{\ell}\right) A \subseteq I=A^{*}\left(I \beta_{\ell}\right)$, then $I \beta_{\ell} \in \operatorname{Sem}(A)$ by Lemma 4.1 and $\Phi$ is well-defined.

On the other hand, given $S \in \operatorname{Sem}(A)$, it is clear that $A^{*} S \unlhd_{\ell} A^{*}$. Now $S A \subseteq A^{*} S$ by Lemma 4.1, hence $A^{*} S$ is actually an ideal of $A^{*}$ and so $\Psi$ is also well-defined.

Now $I \Phi \Psi=A^{*}\left(I \beta_{\ell}\right)=I$ and $S \Psi \Phi=\left(A^{*} S\right) \beta_{\ell}=S$ follows easily from $S$ being a suffix code, hence $\Phi$ and $\Psi$ are mutually inverse bijections. Since $S \leq S^{\prime}$ if and only if $S \Psi \subseteq S^{\prime} \Psi$ holds for all $S, S^{\prime} \in \operatorname{Sem}(A), \Phi$ and $\Psi$ are actually mutually inverse poset isomorphisms. Since $(\mathcal{I}(A), \subseteq)$ is a lattice, so is $(\operatorname{Sem}(A), \leq)$ and so $\Phi$ and $\Psi$ are lattice isomorphisms.

As we will see in Section 7, semaphore codes are related to special right congruences. 


\section{Right congruences on the minimal ideal of $F(g, k)$}

Now fix a nonempty alphabet $A=\left\{a_{1}, \ldots, a_{g}\right\}$ and a positive integer $k$. We remarked in Subsection 2.3 that $A^{\leq k} \backslash\{\varepsilon\}$ is a set of normal forms for $F(g, k)$, the free pro- $\mathbf{D}_{k}$ semigroup on the set $A=\left\{a_{1}, \ldots, a_{g}\right\}$. Moreover, we can identify $A^{k}$ with $\operatorname{ker}(F(g, k))$. Since $F(g, k)$ is generated by $A$, right congruences on $A^{k}$ can be described as equivalence relations $\rho$ satisfying

$$
u \rho v \Rightarrow(u \circ a) \rho(v \circ a)
$$

for every $a \in A$, or equivalently,

$$
u \rho v \Rightarrow\left((u a) \xi_{k}\right) \rho\left((v a) \xi_{k}\right)
$$

for every $a \in A$.

Given $R \subseteq A^{k} \times A^{k}$, we denote by $R^{\sharp}$ the right congruence on $A^{k}$ generated by $R$, i.e. the intersection of all right congruences on $A^{k}$ containing $R$. Let $u, v \in A^{k}$. Then $(u, v) \in R^{\sharp}$ if and only if there exists some finite sequence $w_{0}, \ldots, w_{n} \in A^{k}(n \geq 0)$ such that:

- $w_{0}=u$ and $w_{n}=v$;

- for every $i=1, \ldots, n$, there exist $\left(r_{i}, s_{i}\right) \in R$ and $x_{i} \in A^{*}$ such that $\left\{w_{i-1}, w_{i}\right\}=\left\{r_{i} \circ x_{i}, s_{i} \circ x_{i}\right\}$.

It is easy to see that

$$
\vee \Lambda=(\cup \Lambda)^{\#}
$$

for every $\Lambda \subseteq \mathrm{RC}\left(A^{k}\right)$.

We now relate right congruences on $A^{k}$ with the $k$-reset graphs introduced in Section 3 .

Given $\rho \in \mathrm{RC}\left(A^{k}\right)$, the Cayley graph of $\rho$ is the $A$-graph Cay $(\rho)=\left(A^{k} / \rho, E\right)$ defined by

$$
E=\left\{(u \rho, a,(u \circ a) \rho) \mid u \in A^{k}, a \in A\right\}
$$

where $u \rho$ denotes the congruence class of $u$. In particular, if $\rho$ is the identity relation, then Cay $(\rho)$ is a $k$-dimensional De Bruijn graph on $|A|$ symbols.

Given $\Gamma=(Q, E) \in \mathrm{RG}_{k}(A)$, let $\zeta_{\Gamma}$ be the equivalence relation on $A^{k}$ defined by

$$
u \zeta_{\Gamma} v \text { if } Q u=Q v
$$

Note that

$$
Q\left((u a) \xi_{k}\right)=Q u a
$$

holds for all $u \in A^{k}$ and $a \in A$. Indeed, since $Q u a \subseteq Q\left((u a) \xi_{k}\right)$ and $(u a) \xi_{k}$ is a reset word, we must have equality and (5.1) holds.

Proposition 5.1 Let $A$ be a finite nonempty alphabet and $k \geq 1$. Then

$$
\begin{aligned}
& \Phi:\left(\mathrm{RC}\left(A^{k}\right), \subseteq\right) \rightarrow\left(\mathrm{RG}_{k}(A) / \cong, \leq\right) \quad \text { and } \quad \Psi:\left(\mathrm{RG}_{k}(A) / \cong, \leq\right) \rightarrow\left(\mathrm{RC}\left(A^{k}\right), \subseteq\right) \\
& \rho \mapsto[\mathrm{Cay}(\rho)] \quad \text { and } \quad[\Gamma] \mapsto \zeta_{\Gamma}
\end{aligned}
$$

are mutually inverse lattice isomorphisms. 
Proof. Let $\rho \in \mathrm{RC}\left(A^{k}\right)$. It follows from the definition that Cay $(\rho)$ is deterministic and complete. For all $u, v \in A^{k}$, we have $u \circ v=v$, hence there exists a path

$$
u \rho \stackrel{v}{\longrightarrow}(u \circ v) \rho=v \rho
$$

in $\operatorname{Cay}(\rho)$. It follows that $\operatorname{Cay}(\rho)$ is strongly connected and $A^{k} \subseteq \operatorname{Res}_{k}(\operatorname{Cay}(\rho))$, thus $\operatorname{Cay}(\rho) \in$ $\mathrm{RG}_{k}(A)$ and $\Phi$ is well-defined.

On the other hand, it is clear that $[\Gamma] \Psi$ does not depend on the chosen representative for the isomorphism class $[\Gamma]$.

Let $\Gamma \in \mathrm{RG}_{k}(A)$. Let $(u, v) \in \zeta_{\Gamma}$ and $a \in A$. Then $Q u=Q v$ implies $Q u a=Q v a$ and therefore $(u \circ a, v \circ a) \in \zeta_{\Gamma}$ in view of (5.1). Thus $\zeta_{\Gamma} \in \mathrm{RC}\left(A^{k}\right)$ and so $\Psi$ is well-defined.

Let $\rho \in \operatorname{RC}\left(A^{k}\right)$ and write $\rho^{\prime}=\zeta_{\operatorname{Cay}(\rho)}$. If $Q=A^{k} / \rho$ is the vertex set of $\operatorname{Cay}(\rho)$, then $Q u=\{u \rho\}$ for every $u \in A^{k}$. Hence

$$
u \rho^{\prime} v \Leftrightarrow Q u=Q v \Leftrightarrow u \rho=v \rho
$$

and so $\Phi \Psi=1$.

Conversely, let $\Gamma=(Q, E) \in \mathrm{RG}_{k}(A)$ and let $\Gamma^{\prime}=\operatorname{Cay}\left(\zeta_{\Gamma}\right)$. We show that

$$
\forall q \in Q \exists u_{q} \in A^{k}: Q u_{q}=\{q\} .
$$

We may assume that $|Q|>1$. Since $\Gamma$ is strongly connected, it follows that there exists a loop $q \stackrel{w}{\longrightarrow} q$ in $\Gamma$ with $w \neq \varepsilon$. Replacing $w$ by a proper power if necessary, we may assume that $|w| \geq k$. Hence there exists some $u_{q} \in A^{k}$ such that $q \in Q u_{q}$. Since $u_{q}$ is necessarily a reset word, we get $Q u_{q}=\{q\}$ and so (5.2) holds.

We define a mapping

$$
\begin{aligned}
\theta: Q & \rightarrow A^{k} / \zeta_{\Gamma} . \\
q & \mapsto u_{q} \zeta_{\Gamma}
\end{aligned}
$$

Note that

$$
Q u=Q v \Leftrightarrow u \zeta_{\Gamma}=v \zeta_{\Gamma}
$$

holds for all $u, v \in A^{k}$, hence $\theta$ is well-defined and one-to-one. Since $\Gamma$ is a $k$-reset graph, $\theta$ is also onto. We show that $\theta$ is an isomorphism from $\Gamma$ onto $\operatorname{Cay}\left(\zeta_{\Gamma}\right)$.

Assume that $(p, a, q) \in E$. By (5.1), we get

$$
Q\left(u_{p} \circ a\right)=Q u_{p} a=p a=q=Q u_{q} .
$$

Hence $u_{q} \zeta_{\Gamma}=\left(u_{p} \circ a\right) \zeta_{\Gamma}$ and so there exists an edge $u_{p} \zeta_{\Gamma} \stackrel{a}{\longrightarrow} u_{q} \zeta_{\Gamma}$ in Cay $\left(\zeta_{\Gamma}\right)$.

Conversely, assume that $u_{p} \zeta_{\Gamma} \stackrel{a}{\longrightarrow} u_{q} \zeta_{\Gamma}$ is an edge of Cay $\left(\zeta_{\Gamma}\right)$. Then $u_{q} \zeta_{\Gamma}=\left(u_{p} \circ a\right) \zeta_{\Gamma}$ and so

$$
q=Q u_{q}=Q\left(u_{p} \circ a\right)=Q u_{p} a=p a
$$

by (5.3) and (5.1). Thus $(p, a, q) \in E$ and so $\theta: \Gamma \rightarrow \operatorname{Cay}\left(\zeta_{\Gamma}\right)$ is an isomorphism. Therefore $\Psi \Phi=1$ and so $\Phi$ and $\Psi$ are mutually inverse bijections.

Let $\rho, \rho^{\prime} \in \operatorname{RC}\left(A^{k}\right)$ with $\rho \subseteq \rho^{\prime}$. Then

$$
\begin{aligned}
\theta: A^{k} / \rho & \rightarrow A^{k} / \rho^{\prime} \\
u \rho & \mapsto u \rho^{\prime}
\end{aligned}
$$


is a well-defined surjective map. If $u \rho \stackrel{a}{\longrightarrow}(u \circ a) \rho$ is an edge of Cay $(\rho)$, then $u \rho^{\prime} \stackrel{a}{\longrightarrow}(u \circ a) \rho^{\prime}$ is an edge of $\operatorname{Cay}(\rho)^{\prime}$, hence $\theta$ is a morphism from $\operatorname{Cay}(\rho)$ to $\operatorname{Cay}\left(\rho^{\prime}\right)$ and so $\operatorname{Cay}(\rho) \leq \operatorname{Cay}\left(\rho^{\prime}\right)$. Thus $[\operatorname{Cay}(\rho)] \leq\left[\operatorname{Cay}\left(\rho^{\prime}\right)\right]$ and so $\Phi$ is order-preserving.

Let $\Gamma, \Gamma^{\prime} \in \mathrm{RG}_{k}(A)$ be such that $[\Gamma] \leq\left[\Gamma^{\prime}\right]$. Then there exists a morphism $\theta: \Gamma \rightarrow \Gamma^{\prime}$. Write $\Gamma=(Q, E)$ and $\Gamma^{\prime}=\left(Q^{\prime}, E^{\prime}\right)$. Suppose that $(u, v) \in \zeta_{\Gamma}$. Then $Q u=Q v=\{q\}$ for some $q \in Q$. Hence $q \theta \in Q^{\prime} u \cap Q^{\prime} v$. Since $\Gamma^{\prime}$ is a $k$-reset graph, we get $Q^{\prime} u=\{q \theta\}=Q^{\prime} v$ and so $(u, v) \in \zeta_{\Gamma^{\prime}}$. Therefore $\Psi$ is order-preserving.

Since $\Phi$ and $\Psi$ are mutually inverse order-preserving mappings, they are isomorphisms of posets. Since $\left(\mathrm{RC}\left(A^{k}\right), \subseteq\right)$ is a lattice, then $\left(\operatorname{RG}_{k}(A), \leq\right)$ is also a lattice, and $\Phi$ and $\Psi$ are mutually inverse lattice isomorphisms.

\section{$6 \quad$ Lattice-theoretic properties}

We discuss in this section the lattice-theoretic properties of the lattice $\operatorname{RC}\left(A^{k}\right)$.

We recall some well-known notions from lattice theory. Let $L$ be a (finite) lattice with bottom element $B$ and top element $T$. Given $a, b \in L$, we say that $b$ covers $a$ if $a<b$ and there is no $c \in L$ such that $a<c<b$. If $a$ covers the bottom $B$, we say that $a$ is an atom.

The lattice $L$ is said to be:

- modular if it has no sublattice of the form

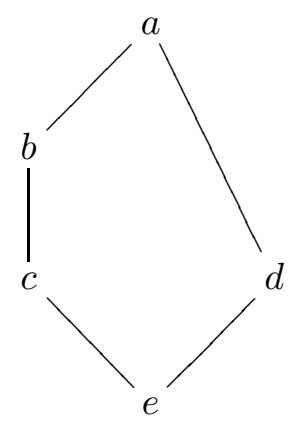

- semimodular if it has no sublattice of the form (6.1) with $d$ covering $e$;

- atomistic if every element of $L$ is a join of atoms ( $B$ being the join of the empty set).

Proposition 6.1 Let $A$ be a nonempty set and $k \geq 1$. Then $\operatorname{RC}\left(A^{k}\right)$ is semimodular.

Proof. It suffices to show that $\operatorname{RC}\left(A^{k}\right)$ has no sublattice of the form

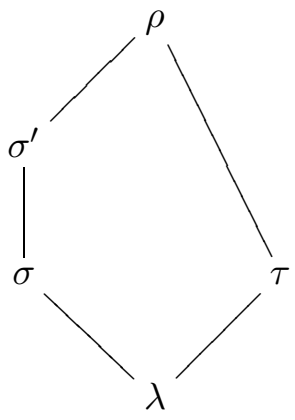


with $\tau$ covering $\lambda$ in $\operatorname{RC}\left(A^{k}\right)$.

Suppose it does. Given $x, y \in A^{*}$, let $\operatorname{lcs}(x, y)$ denote the longest common suffix of $x$ and $y$. If $x, y \in A^{k}$ are distinct, then $|\operatorname{lcs}(x, y)|<k$ and so

$$
|\operatorname{lcs}(x \circ a, y \circ a)|>|\operatorname{lcs}(x, y)|
$$

for every $a \in A$.

Let $(u, v) \in \tau \backslash \lambda$ with $|\operatorname{lcs}(u, v)|$ maximal. For every $a \in A$, we have

$$
(u \circ a, v \circ a) \in\{(u, v)\}^{\sharp} \subseteq \tau .
$$

In view of $(6.2)$, and by maximality of $|\operatorname{lcs}(u, v)|$, we get

$$
(u \circ a, v \circ a) \in \lambda \text {. }
$$

Note also that

$$
\lambda \subset(\lambda \cup\{(u, v)\})^{\sharp} \subseteq \tau
$$

yields

$$
\tau=(\lambda \cup\{(u, v)\})^{\sharp}
$$

since $\tau$ covers $\lambda$.

Let $(y, z) \in \sigma^{\prime} \backslash \sigma$. Then (6.4) yields

$$
(y, z) \in \rho=(\sigma \vee \tau)=\left(\sigma \cup(\lambda \cup\{(u, v)\})^{\sharp}\right)^{\sharp}=(\sigma \cup\{(u, v)\})^{\sharp}
$$

and so there exists some finite sequence $w_{0}, \ldots, w_{n} \in A^{k}$ such that:

- $w_{0}=y$ and $w_{n}=z$;

- for every $i=1, \ldots, n$, there exist $\left(r_{i}, s_{i}\right) \in \sigma \cup\{(u, v)\}$ and $x_{i} \in A^{*}$ such that $\left\{w_{i-1}, w_{i}\right\}=$ $\left\{r_{i} \circ x_{i}, s_{i} \circ x_{i}\right\}$.

Now by (6.3) we may assume that $x_{i}=\varepsilon$ whenever $\left(r_{i}, s_{i}\right)=(u, v)$. Since we may assume that the $w_{i}$ are all distinct, the relation $(u, v)$ is used at most once, indeed exactly once since $(y, z) \notin \sigma$ and $\left(r_{i}, s_{i}\right) \in \sigma$ implies $\left(r_{i} \circ x_{i}, s_{i} \circ x_{i}\right) \in \sigma$. We may assume without loss of generality that $u=w_{j-1}$ and $v=w_{j}$ for some $j \in\{1, \ldots, n\}$. Hence

$$
y=w_{0} \sigma w_{j-1}=u, \quad v=w_{j} \sigma w_{n}=z
$$

and so

$$
u=w_{j-1} \sigma^{\prime} y \sigma^{\prime} z \sigma^{\prime} w_{j}=v .
$$

It follows that $\lambda \cup\{(u, v)\} \subseteq \sigma^{\prime}$. By (6.4), we get $\tau \subseteq \sigma^{\prime}$, a contradiction. Therefore $\operatorname{RC}\left(A^{k}\right)$ is semimodular.

Since a semimodular lattice of finite height (i.e. the length of chains is bounded) satisfies the Jordan-Dedekind condition (i.e. all maximal chains have the same length), we immediately obtain:

Corollary 6.2 Let $A$ be a nonempty set and $k \geq 1$. Then $\operatorname{RC}\left(A^{k}\right)$ satisfies the Jordan-Dedekind condition.

We show next that we cannot replace semimodular by modular in Proposition 6.1.

Proposition 6.3 Let $k \geq 1$ and let $A$ be a set with $|A| \geq 4$. Then $\operatorname{RC}\left(A^{k}\right)$ is not modular. 
Proof. Let $a, b, c, d \in A$ be distinct. Let $\lambda$ be the identity relation on $A^{k}$ and let

$$
\begin{aligned}
& \sigma=\lambda \cup\left\{a^{k}, b a^{k-1}\right\}^{2} ; \\
& \sigma^{\prime}=\lambda \cup\left\{a^{k}, b a^{k-1}\right\}^{2} \cup\left\{c a^{k-1}, d a^{k-1}\right\}^{2} ; \\
& \tau=\lambda \cup\left\{a^{k}, d a^{k-1}\right\}^{2} \cup\left\{b a^{k-1}, c a^{k-1}\right\}^{2} ; \\
& \rho=\lambda \cup\left\{a^{k}, b a^{k-1}, c a^{k-1}, d a^{k-1}\right\}^{2} .
\end{aligned}
$$

It is routine to check that all the above relations are right congruences on $A^{k}$. Moreover,

$$
\begin{gathered}
\lambda \subset \sigma \subset \sigma^{\prime} \subset \rho, \quad \lambda \subset \tau \subset \rho, \\
\sigma^{\prime} \cap \tau=\lambda, \quad(\sigma \vee \tau)=\rho,
\end{gathered}
$$

hence

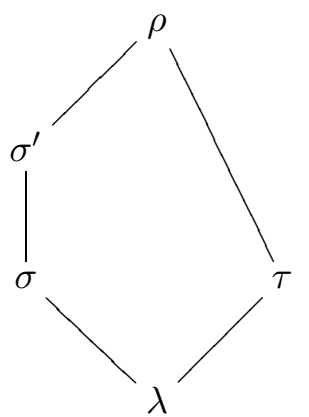

is a sublattice of $\mathrm{RC}\left(A^{k}\right)$ and so $\mathrm{RC}\left(A^{k}\right)$ is not modular.

We can also show that $\mathrm{RC}\left(A^{k}\right)$ can only be atomistic in trivial cases:

Proposition 6.4 Let $k \geq 2$ and let $A$ be a set with $|A| \geq 2$. Then $\mathrm{RC}\left(A^{k}\right)$ is not atomistic.

Proof. Let $\lambda$ be the identity relation on $A^{k}$. Let $a, b \in A$ be distinct and let

$$
\begin{aligned}
& \sigma=\lambda \cup\left\{a^{k}, b^{2} a^{k-2}, b a^{k-1}\right\}^{2} \cup\left\{a^{k-1} b, b a^{k-2} b\right\}^{2} ; \\
& \tau=\lambda \cup\left\{a^{k}, b a^{k-1}\right\}^{2} \cup\left\{a^{k-1} b, b a^{k-2} b\right\}^{2} .
\end{aligned}
$$

It is routine to check that $\sigma, \tau \in \operatorname{RC}\left(A^{k}\right)$. Moreover, $\lambda \subset \tau \subset \sigma$. We show that

$$
\sigma=\left\{\left(x a^{k-1}, b^{2} a^{k-2}\right)\right\}^{\sharp}
$$

for every $x \in\{a, b\}$. Indeed, let $\eta=\left\{\left(x a^{k-1}, b^{2} a^{k-2}\right)\right\}^{\sharp}$. Then $\left(x a^{k-1}, b^{2} a^{k-2}\right) \in \eta$ yields $\left(a^{k}, b a^{k-1}\right) \in$ $\eta$ and so $\left\{a^{k}, b^{2} a^{k-2}, b a^{k-1}\right\}^{2} \subseteq \eta$. Finally, $\left(x a^{k-1}, b^{2} a^{k-2}\right) \in \eta$ yields $\left(a^{k-1} b, b a^{k-2} b\right) \in \eta$ and so

$$
\sigma \subseteq\left\{\left(x a^{k-1}, b^{2} a^{k-2}\right)\right\}^{\sharp} .
$$

Since $\left(x a^{k-1}, b^{2} a^{k-2}\right) \in \sigma$ for $x \in\{a, b\},(6.5)$ holds.

Now we claim that $\tau$ is the unique element of $\operatorname{RC}\left(A^{k}\right)$ covered by $\sigma$. Indeed, assume that $\rho \subset \sigma$. In view of (6.5), we have $\left(a^{k}, b^{2} a^{k-2}\right) \notin \rho$ and $\left(b a^{k-1}, b^{2} a^{k-2}\right) \notin \rho$. Hence $\rho \subseteq \tau$. Since $\sigma$ is not an atom, it follows that

$$
\alpha \leq \sigma \text { if and only if } \alpha \leq \tau
$$

for every atom $\alpha$ of $\operatorname{RC}\left(A^{k}\right)$. Thus $\sigma$ cannot be expressed as a join of atoms and so $\mathrm{RC}\left(A^{k}\right)$ is not atomistic. 


\section{Special right congruences on $A^{k}$}

To avoid trivial cases, we assume throughout this section that $A$ is a finite alphabet containing at least two elements. We define

$$
\begin{gathered}
\mathcal{I}_{k}(A)=\left\{I \unlhd A^{*} \mid A^{k} \subset I\right\}, \\
\mathcal{L}_{k}(A)=\left\{L \unlhd_{\ell} A^{*} \mid A^{k} \subset L\right\} .
\end{gathered}
$$

If we order $\mathcal{I}_{k}(A)$ (or $\mathcal{L}_{k}(A)$ ) by inclusion, we get a finite (distributive) lattice where meet and join are given by

$$
(I \wedge J)=I \cap J, \quad(I \vee J)=I \cup J
$$

The top element is $A^{*}$ and the bottom element is $A^{k} A^{*}$.

Given $L \in \mathcal{L}_{k}(A)$, we define a relation $\tau_{L}$ on $A^{k}$ by:

$u \tau_{L} v$ if $u$ and $v$ have a common suffix in $L$.

Lemma 7.1 Let $L \in \mathcal{L}_{k}(A)$. Then $\tau_{L}$ is an equivalence relation on $A^{k}$.

Proof. It is immediate that $\tau_{L}$ is symmetric. Since $A^{k} \subseteq L$, it is reflexive. Assume now that $u, v, w \in A^{k}$ and $x, y \in L$ are such that $x \leq_{s} u, v$ and $y \leq_{s} v, w$. Since $x$ and $y$ are both suffixes of $v$, one of them is a suffix of the other. Hence either $x \leq_{s} u, w$ or $y \leq_{s} u, w$. Therefore $\tau_{L}$ is transitive.

Being a right congruence turns out to be a special case:

Proposition 7.2 Let $L \in \mathcal{L}_{k}(A)$. Then the following conditions are equivalent:

(i) $\tau_{L} \in \mathrm{RC}\left(A^{k}\right)$;

(ii) $L \in \mathcal{I}_{k}(A)$;

(iii) $\left(L \beta_{\ell}\right) A \subseteq A^{*}\left(L \beta_{\ell}\right)$;

(iv) $L \beta_{\ell}$ is a semaphore code.

Proof. (i) $\Rightarrow$ (iii). Let $u \in L \beta_{\ell}$ and $a \in A$. Since $A^{*}\left(L \beta_{\ell}\right)=L \supset A^{k}$, we may assume that $|u|<k-1$. Let $b \in A \backslash\{a\}$ and write $m=k-|u|$. Then $\left(a^{m} u, b^{m} u\right) \in \tau_{L}$, hence

$$
\left(a^{m-1} u a, b^{m-1} u a\right)=\left(a^{m} u \circ a, b^{m} u \circ a\right) \in \tau_{L} .
$$

It follows that $a^{m-1} u a$ and $b^{m-1} u a$ must share a suffix in $L$, and so $u a$ itself must have a suffix in L. Thus

$$
\left(L \beta_{\ell}\right) A \subseteq A^{*} L=L=A^{*}\left(L \beta_{\ell}\right)
$$

(iii) $\Rightarrow$ (ii). We have

$$
L A=A^{*}\left(L \beta_{\ell}\right) A \subseteq A^{*}\left(L \beta_{\ell}\right)=L .
$$

It follows that $L A^{*} \subseteq L$. Since $L \in \mathcal{L}_{k}(A)$, we get $L \in \mathcal{I}_{k}(A)$.

(ii) $\Rightarrow$ (i). By Lemma 7.1, $\tau_{L}$ is an equivalence relation. Let $u, v \in A^{k}$ be such that $u \tau_{L} v$. Then $w \leq_{s} u, v$ for some $w \in L$. We may assume that $|w|<k$. Let $a \in A$. Since $L \unlhd A^{*}$, we have $w a \in L$. Since $|w|<k$, it follows that $w a$ is a common suffix of $u \circ a$ and $v \circ a$. Therefore $(u \circ a) \tau_{L}(v \circ a)$ and we are done.

(iii) $\Leftrightarrow$ (iv). This follows from Lemma 4.1 , since $L \beta_{\ell}$ is always a suffix code. 
Note that we can easily produce examples of $L \in \mathcal{L}_{k}(A) \backslash \mathcal{I}_{k}(A)$ :

Example 7.3 Let $A=\{a, b\}, k=3$ and $L=A^{*} b \cup A^{+} A a$. Then $L \in \mathcal{L}_{k}(A)$ but $\tau_{L} \notin \operatorname{RC}\left(A^{k}\right)$.

Indeed, $b \in L$ but $b a \notin L$, hence $L \notin \mathcal{I}_{k}(A)$ and so $\tau_{L} \notin \operatorname{RC}\left(A^{k}\right)$ by Proposition 7.2. Note that in this case $\beta_{\ell}=\left\{b, a^{3}, b a^{2}, a b a, b^{2} a\right\}$.

Inclusion among left ideals determines inclusion for the equivalence relations $\tau_{L}$ :

Lemma 7.4 Let $|A|>1$ and $L, L^{\prime} \in \mathcal{L}_{k}(A)$. Then

$$
\tau_{L} \subseteq \tau_{L^{\prime}} \Leftrightarrow L \subseteq L^{\prime}
$$

Proof. Assume that $L \subseteq L^{\prime}$. Let $(u, v) \in \tau_{L}$. Then $u$ and $v$ share a common suffix in $L$ and therefore in $L^{\prime}$. Thus $(u, v) \in \tau_{L^{\prime}}$.

Assume now that $L \nsubseteq L^{\prime}$. Let $w \in L \backslash L^{\prime}$ have minimum length. Since $A^{k} \subseteq L^{\prime}$, we have $|w|<k$. Let $n=k-|w|$. Fix $a, b \in A$ distinct and take $(u, v)=\left(a^{n} w, b^{n} w\right) \in A^{k} \times A^{k}$. Since $w \in L$, we have $(u, v) \in \tau_{L}$. Now $w$ is the longest common suffix of $u$ and $v$. Since $w \notin L^{\prime}$, it follows that $(u, v) \notin \tau_{L^{\prime}}$.

Note that Lemma 7.4 does not hold for $|A|=1$, since $\left|A^{k}\right|=1$.

Definition 7.5 We say that $\rho \in \mathrm{RC}\left(A^{k}\right)$ is a special right congruence on $A^{k}$ if $\rho=\tau_{I}$ for some $I \in \mathcal{I}_{k}(A)$. In view of Proposition 7.2, this is equivalent to say that $\rho=\tau_{A^{*} S}$ for some semaphore code $S$ on $A$ such that $A^{k} \subset A^{*} S$. We denote by $\operatorname{SRC}\left(A^{k}\right)$ the set of all special right congruences on $A^{k}$.

Note that not every semaphore code $S$ satisfies the condition $A^{k} \subset A^{*} S$. However, it is easy to derive a semaphore code from $S$ that does by considering

$$
S^{\prime}=\left(S \cap A^{\leq k}\right) \cup\left(A^{k} \backslash A^{*} S\right) .
$$

$S^{\prime}$ is a suffix code since the elements in $S \cap A^{\leq k}$ are incomparable in suffix order since $S$ is a suffix code, and by construction any element in $A^{k} \backslash A^{*} S$ is incomparable with the elements in $S \cap A^{\leq k}$ and vice versa. Furthermore, $A^{k} \subset A^{*} S^{\prime} \supseteq A^{*} S$ and $S A \subseteq A^{*} S$ by Lemma 4.1. Thus $S^{\prime} A \subseteq A^{*} S^{\prime}$ and so by Lemma $4.1 S^{\prime}$ is a semaphore code.

Proposition 7.6 Let $|A|>1$. Then:

(i) $\tau_{I \cap J}=\tau_{I} \cap \tau_{J}$ and $\tau_{I \cup J}=\tau_{I} \cup \tau_{J}$ for all $I, J \in \mathcal{I}_{k}(A)$;

(ii) $\operatorname{SRC}\left(A^{k}\right)$ is a full sublattice of $\mathrm{RC}\left(A^{k}\right)$;

(iii) the mapping

$$
\begin{aligned}
\mathcal{I}_{k}(A) & \rightarrow \operatorname{SRC}\left(A^{k}\right) \\
I & \mapsto \tau_{I}
\end{aligned}
$$

is a lattice isomorphism.

Proof. (i) By Lemma 7.4, we have $\tau_{I \cap J} \subseteq \tau_{I} \cap \tau_{J}$ and $\tau_{I} \cup \tau_{J} \subseteq \tau_{I \cup J}$.

Let $(u, v) \in \tau_{I} \cap \tau_{J}$. Then there exist $x \in I$ and $y \in J$ such that $x \leq_{s} u, v$ and $y \leq_{s} u, v$. Since $x$ and $y$ are both suffixes of the same word, one of them is a suffix of the other, say $x \leq_{s} y$. Then $y \in I \cap J$ and so $(u, v) \in \tau_{I \cap J}$. Thus $\tau_{I \cap J}=\tau_{I} \cap \tau_{J}$. 
Assume now that $(u, v) \in \tau_{I \cup J}$. Then there exists some $x \in I \cup J$ such that $x \leq_{s} u, v$. If $x \in I$, then $(u, v) \in \tau_{I}$, otherwise $(u, v) \in \tau_{J}$. Therefore $\tau_{I \cup J}=\tau_{I} \cup \tau_{J}$.

(ii) Let $I, J \in \mathcal{I}_{k}(A)$. By part (i), $\tau_{I \cap J}$ is the meet of $\tau_{I}$ and $\tau_{J}$ in both $\operatorname{RC}\left(A^{k}\right)$ and $\operatorname{SRC}\left(A^{k}\right)$. And $\tau_{I \cup J}$ is the join of $\tau_{I}$ and $\tau_{J}$ in both $\operatorname{RC}\left(A^{k}\right)$ and $\operatorname{SRC}\left(A^{k}\right)$.

Finally, $\tau_{A^{k} A^{*}}$ is the identity relation and therefore the bottom element of both lattices. And $\tau_{A^{*}}$ is the universal relation and therefore the top element of both lattices.

(iii) This follows from Lemma 7.4.

Given $\rho \in \mathrm{RC}\left(A^{k}\right)$ and $C \in A^{k} / \rho$, we denote by $\operatorname{lcs}(C)$ the longest common suffix of all words in $C$. We define

$$
\Lambda_{\rho}=\left\{\operatorname{lcs}(C) \mid C \in A^{k} / \rho\right\} \quad \text { and } \quad \Lambda_{\rho}^{\prime}=\{\operatorname{lcs}(u, v) \mid(u, v) \in \rho\} .
$$

Lemma 7.7 Let $\rho \in \operatorname{RC}\left(A^{k}\right)$. Then $A^{*} \Lambda_{\rho}=A^{*} \Lambda_{\rho}^{\prime} \in \mathcal{I}_{k}(A)$.

Proof. Let $C \in A^{k} / \rho$ and let $w=\operatorname{lcs}(C)$. If $|w|=k$, then $w=\operatorname{lcs}(w, w)$. If $|w|<k$, then by maximality of $w$ there exist $a, b \in A$ distinct and $u, v \in A^{*}$ such that uaw, vbw $\in C$. Thus $w=\operatorname{lcs}(u a w, v b w)$ and so

$$
\Lambda_{\rho} \subseteq \Lambda_{\rho}^{\prime}
$$

Therefore $A^{*} \Lambda_{\rho} \subseteq A^{*} \Lambda_{\rho}^{\prime}$.

Conversely, let $(u, v) \in \rho$. Then $\operatorname{lcs}(u \rho)$ is a suffix of $\operatorname{lcs}(u, v)$, hence $\Lambda_{\rho}^{\prime} \subseteq A^{*} \Lambda_{\rho}$ and so $A^{*} \Lambda_{\rho}=$ $A^{*} \Lambda_{\rho}^{\prime}$.

Clearly, $A^{*} \Lambda_{\rho}^{\prime} \unlhd_{\ell} A^{*}$. Since $u=\operatorname{lcs}(u, u)$ for every $u \in A^{k}$, we have $A^{k} \subseteq \Lambda_{\rho}^{\prime}$. Hence it suffices to show that $\left(\Lambda_{\rho}^{\prime}\right) A \subseteq A^{*} \Lambda_{\rho}^{\prime}$.

Let $(u, v) \in \rho$ and $a \in A$. We must show that $(\operatorname{lcs}(u, v)) a \in A^{*} \Lambda_{\rho}^{\prime}$. Since $A^{k} \subseteq \Lambda_{\rho}^{\prime}$, we may assume that $|\operatorname{lcs}(u, v)|<k-1$. Then $(\operatorname{lcs}(u, v)) a=\operatorname{lcs}(u \circ a, v \circ a)$. Since $(u \circ a, v \circ a) \in \rho$, we get $(\operatorname{lcs}(u, v)) a \in \Lambda_{\rho}^{\prime}$ and we are done.

Given $\rho \in \mathrm{RC}\left(A^{k}\right)$, we write

$$
\operatorname{Res}(\rho)=\operatorname{Res}(\operatorname{Cay}(\rho)) .
$$

We refer to the elements of $\operatorname{Res}(\rho)$ as the resets of $\rho$.

Lemma 7.8 Let $\rho \in \mathrm{RC}\left(A^{k}\right)$. Then:

(i) $\operatorname{Res}(\rho)=\left\{w \in A^{*} \mid u \rho v\right.$ for all $\left.u, v \in A^{k} \cap\left(A^{*} w\right)\right\}$;

(ii) $\operatorname{Res}(\rho) \in \mathcal{I}_{k}(A)$.

Proof. (i) Let $w \in \operatorname{Res}(\rho)$ and suppose that $u=u^{\prime} w \in A^{k}, v=v^{\prime} w \in A^{k}$. Since $w \in \operatorname{Res}(\rho)$, we have paths

$$
p \stackrel{u^{\prime}}{\longrightarrow} p^{\prime} \stackrel{w}{\longrightarrow} r, \quad q \stackrel{v^{\prime}}{\longrightarrow} q^{\prime} \stackrel{w}{\longrightarrow} r
$$

in $\operatorname{Cay}(\rho)$. It follows from the definition of $\operatorname{Cay}(\rho)$ that

$$
u \rho=\left(u^{\prime} w\right) \rho=r=\left(v^{\prime} w\right) \rho=v \rho,
$$

hence the direct inclusion holds. 
To prove the opposite inclusion, we suppose that $w \in A^{*} \backslash \operatorname{Res}(\rho)$. Then there exist paths

$$
p^{\prime} \stackrel{w}{\longrightarrow} p, \quad q^{\prime} \stackrel{w}{\longrightarrow} q
$$

in Cay $(\rho)$ with $p \neq q$. If $w$ has a suffix $w^{\prime}$ of length $k$, then every path labeled by $w$ ends necessarily in $w^{\prime} \rho$, hence we must have $|w|<k$. Since Cay $(\rho)$ is strongly connected by Proposition 5.1 , there exist paths

$$
p^{\prime \prime} \stackrel{x}{\longrightarrow} p^{\prime}, \quad q^{\prime \prime} \stackrel{y}{\longrightarrow} q^{\prime}
$$

in $\operatorname{Cay}(\rho)$ with $|x w|=|y w|=k$. But then

$$
(x w) \rho=p \neq q=(y w) \rho
$$

and we are done.

(ii) It is immediate that $\operatorname{Res}(\rho) \unlhd A^{*}$. Since every path in Cay $(\rho)$ labeled by $w \in A^{k}$ ends necessarily in $w \rho$, we have $A^{k} \subseteq \operatorname{Res}(\rho)$ and so $\operatorname{Res}(\rho) \in \mathcal{I}_{k}(A)$.

We can now compare a right congruence with a special right congruence:

Proposition 7.9 Let $|A|>1, \rho \in \mathrm{RC}\left(A^{k}\right)$ and $I \in \mathcal{I}_{k}(A)$. Then:

(i) $\rho \subseteq \tau_{I} \Leftrightarrow \Lambda_{\rho} \subseteq I \Leftrightarrow \Lambda_{\rho}^{\prime} \subseteq I$;

(ii) $\tau_{I} \subseteq \rho \Leftrightarrow I \subseteq \operatorname{Res}(\rho)$.

Proof. (i) Assume that $\rho \subseteq \tau_{I}$. Let $(u, v) \in \rho$. Then $u$ and $v$ have a common suffix in $I$, hence $\operatorname{lcs}(u, v)$ has a suffix in $I$ and so $\Lambda_{\rho}^{\prime} \subseteq A^{*} I=I$.

By (7.3), $\Lambda_{\rho}^{\prime} \subseteq I$ implies $\Lambda_{\rho} \subseteq I$.

Finally, assume that $\Lambda_{\rho} \subseteq I$. Let $(u, v) \in \rho$ and write $w=\operatorname{lcs}(u \rho) \in \Lambda_{\rho} \subseteq I$. Since $w$ is a suffix of both $u$ and $v$, we get $(u, v) \in \tau_{I}$. Thus $\rho \subseteq \tau_{I}$ as required.

(ii) Assume that $\tau_{I} \subseteq \rho$. Let $w \in I$ and let $u, v \in A^{k} \cap\left(A^{*} w\right)$. Since $u, v$ have a common suffix in $I$, we get $(u, v) \in \tau_{I} \subseteq \rho$. Thus $w \in \operatorname{Res}(\rho)$ by Lemma 7.8(i) and so $I \subseteq \operatorname{Res}(\rho)$.

Conversely, assume that $I \subseteq \operatorname{Res}(\rho)$. Let $(u, v) \in \tau_{I}$. Then we may write $u=u^{\prime} w, v=v^{\prime} w$ with $w \in I \subseteq \operatorname{Res}(\rho)$. Since $u, v \in A^{k} \cap\left(A^{*} w\right)$, it follows from Lemma 7.8(i) that $(u, v) \in \rho$ and so $\tau_{I} \subseteq \rho$.

We can now prove several equivalent characterizations of special right congruences:

Proposition 7.10 Let $|A|>1$ and $\rho \in \mathrm{RC}\left(A^{k}\right)$. Then the following conditions are equivalent:

(i) $\rho \in \operatorname{SRC}\left(A^{k}\right)$;

(ii) lcs : $A^{k} / \rho \rightarrow A^{\leq k}$ is injective and $\Lambda_{\rho}$ is a suffix code;

(iii) $\rho=\tau_{A^{*} \Lambda_{\rho}}$;

(iv) $\rho=\tau_{A^{*} \Lambda_{\rho}^{\prime}}$;

(v) $\rho=\tau_{\operatorname{Res}(\rho)} ;$

(vi) $\rho=\tau_{L}^{\sharp}$ for some $L \in \mathcal{L}_{k}(A)$; 
(vii) $\Lambda_{\rho} \subseteq \operatorname{Res}(\rho)$;

(viii) $\Lambda_{\rho}^{\prime} \subseteq \operatorname{Res}(\rho)$;

(ix) whenever

$$
p \stackrel{a w}{\longrightarrow} q, \quad p^{\prime} \stackrel{b w}{\longrightarrow} q, \quad p^{\prime \prime} \stackrel{w}{\longrightarrow} r
$$

are paths in $\operatorname{Cay}(\rho)$ with $a, b \in A$ distinct, then $q=r$.

Proof. (i) $\Rightarrow$ (ii). We start by proving that

$$
\operatorname{lcs}\left(u \tau_{I}\right) \in I
$$

for all $I \in \mathcal{I}_{k}(A)$ and $u \in A^{k}$.

Indeed, for every $w \in u \tau_{I}$, there exists some $w^{\prime} \in I$ such that $w^{\prime} \leq_{s} u, w$. Let $z$ be the shortest suffix among the $w^{\prime}$. Then $z \in I$ and $z \leq_{s} w$ for every $w \in u \tau_{I}$, hence $z \leq_{s} \operatorname{lcs}\left(u \tau_{I}\right)$. Since $I \unlhd A^{*}$, it follows that $\operatorname{lcs}\left(u \tau_{I}\right) \in I$ and so (7.5) holds.

Assume that $\rho=\tau_{I}$ for some $I \in \mathcal{I}_{k}(A)$. We prove that

$$
\operatorname{lcs}(u \rho) \leq_{s} \operatorname{lcs}(v \rho) \Rightarrow(u, v) \in \rho
$$

holds for all $u, v \in A^{k}$. Assume that $\operatorname{lcs}(u \rho) \leq_{s} \operatorname{lcs}(v \rho)$. Since $\operatorname{lcs}(u \rho) \leq_{s} u$ and $\operatorname{lcs}(v \rho) \leq_{s} v$, it follows that $\operatorname{lcs}(u \rho)$ is a suffix of both $u$ and $v$. Now (7.5) yields $\operatorname{lcs}(u \rho)=\operatorname{lcs}\left(u \tau_{I}\right) \in I$ and so $u, v$ have a common suffix in $I$. Therefore $(u, v) \in \tau_{I}=\rho$ and (7.6) holds.

Now (ii) follows from (7.6).

(ii) $\Rightarrow$ (iii). Write $I=A^{*} \Lambda_{\rho}$. If $(u, v) \in \rho$, then $\operatorname{lcs}(u \rho) \in \Lambda_{\rho} \subseteq I$ is a suffix of both $u$ and $v$, hence $(u, v) \in \tau_{I}$.

Conversely, let $(u, v) \in \tau_{I}$. Then there exists some $w \in \Lambda_{\rho}$ such that $w \leq_{s} u, v$. Suppose that $\operatorname{lcs}(u \rho) \neq w$. Then $\operatorname{lcs}(u \rho)<_{s} w$ or $w<_{s} \operatorname{lcs}(u \rho)$, contradicting $\Lambda_{\rho}$ being a suffix code. Hence $\operatorname{lcs}(u \rho)=w$. Similarly, $\operatorname{lcs}(v \rho)=w$. Since $\operatorname{lcs}: A^{k} / \rho \rightarrow A^{\leq k}$ is injective, we get $u \rho=v \rho$. Thus $\rho=\tau_{I}$.

(iii) $\Leftrightarrow$ (iv). This follows from Lemma 7.7.

(iii) $\Rightarrow$ (vi). Write $L=A^{*} \Lambda_{\rho}$. By (iii), we have $\tau_{L}^{\sharp}=\rho^{\sharp}=\rho$. Since $L \in \mathcal{L}_{k}(A)$ by Lemma 7.7, (vi) holds.

(vi) $\Rightarrow(\mathrm{i})$. Let $I=L A^{*} \in \mathcal{I}_{k}(A)$. Since $L \subseteq I$, it follows from Lemma 7.4 that $\tau_{L} \subseteq \tau_{I}$, hence

$$
\rho=\tau_{L}^{\sharp} \subseteq \tau_{I}^{\sharp}=\tau_{I}
$$

by Proposition 7.2 .

Now assume that $(u, v) \in \tau_{I}$. Then there exist factorizations $u=u^{\prime} w$ and $v=v^{\prime} w$ with $w \in I$. Write $w=z w^{\prime}$ with $z \in L$. Then $\left(w^{\prime} u^{\prime} z, w^{\prime} v^{\prime} z\right) \in \tau_{L}$ and so

$$
(u, v)=\left(u^{\prime} w, v^{\prime} w\right)=\left(u^{\prime} z w^{\prime}, v^{\prime} z w^{\prime}\right)=\left(w^{\prime} u^{\prime} z \circ w^{\prime}, w^{\prime} v^{\prime} z \circ w^{\prime}\right) \in \tau_{L}^{\sharp}=\rho .
$$

Thus $\tau_{I} \subseteq \rho$ as required.

(i) $\Rightarrow(\mathrm{v})$. If $\rho=\tau_{I}$ for some $I \in \mathcal{I}_{k}(A)$, then $I \subseteq \operatorname{Res}(\rho)$ by Proposition 7.9(ii). Since $\operatorname{Res}(\rho) \in \mathcal{I}_{k}(A)$ by Lemma 7.8(ii), then Proposition 7.9(ii) also yields

$$
\tau_{\operatorname{Res}(\rho)} \subseteq \rho=\tau_{I},
$$


hence $\operatorname{Res}(\rho) \subseteq I$ by Lemma 7.4. Therefore $I=\operatorname{Res}(\rho)$.

(v) $\Rightarrow$ (vii) $\Leftrightarrow$ (viii). By Lemma 7.8(ii), $\operatorname{Res}(\rho) \in \mathcal{I}_{k}(A)$. Now we apply Proposition 7.9(i).

(viii) $\Rightarrow$ (i). We have $A^{*} \Lambda_{\rho}^{\prime}, \operatorname{Res}(\rho) \in \mathcal{I}_{k}(A)$ by Lemmas 7.7 and 7.8(ii). It follows from Proposition 7.9 that

$$
\tau_{\operatorname{Res}(\rho)} \subseteq \rho \subseteq \tau_{A^{*} \Lambda_{\rho}^{\prime}}
$$

Since $\Lambda_{\rho}^{\prime} \subseteq \operatorname{Res}(\rho)$ yields $A^{*} \Lambda_{\rho}^{\prime} \subseteq \operatorname{Res}(\rho)$ and therefore $\tau_{A^{*} \Lambda_{\rho}^{\prime}} \subseteq \tau_{\operatorname{Res}(\rho)}$ by Lemma 7.4 , we get $\rho=\tau_{\operatorname{Res}(\rho)} \in \operatorname{SRC}\left(A^{k}\right)$.

(viii) $\Rightarrow$ (ix). Consider the paths in (7.4). Since $A^{k} \subseteq \operatorname{Res}(\rho)$ by Lemma 7.8(ii), we may assume that $|w|<k$. Since $\operatorname{Cay}(\rho)$ is strongly connected, there exist paths

$$
s \stackrel{x}{\longrightarrow} p, \quad s^{\prime} \stackrel{x^{\prime}}{\longrightarrow} p^{\prime}
$$

such that $x a w, x^{\prime} b w \in A^{k}$. Hence

$$
w=\operatorname{lcs}\left(x a w, x^{\prime} b w\right) \in \Lambda_{\rho}^{\prime} \subseteq \operatorname{Res}(\rho)
$$

and so $q=r$.

(ix) $\Rightarrow$ (viii). Let $w \in \Lambda_{\rho}^{\prime}$. Since $A^{k} \subseteq \operatorname{Res}(\rho)$ by Lemma 7.8(ii), we may assume that $|w|<k$. Then $w=\operatorname{lcs}(u, v)$ for some distinct $\rho$-equivalent $u, v \in A^{k}$. Hence we may write $u=u^{\prime} a w$ and $v=v^{\prime} b w$ with $a, b \in A$ distinct. Since $u \rho=v \rho$, it follows that there exist in Cay $(\rho)$ paths of the form

$$
s \stackrel{u^{\prime}}{\longrightarrow} p \stackrel{a w}{\longrightarrow} u \rho, \quad s^{\prime} \stackrel{v^{\prime}}{\longrightarrow} p^{\prime} \stackrel{b w}{\longrightarrow} v \rho .
$$

Now (ix) implies that $w \in \operatorname{Res}(\rho)$.

Corollary 7.11 If $\rho \in \operatorname{SRC}\left(A^{k}\right)$ with $|A|>1$, then $\Lambda_{\rho}$ is a semaphore code.

Proof. By Proposition 7.10(ii), $\Lambda_{\rho}$ is a suffix code. Furthermore, by Lemma 7.7 we have $A^{*} \Lambda_{\rho} \in$ $\mathcal{I}_{k}(A)$, which in turn implies by Proposition 7.2 that $\left(A^{*} \Lambda_{\rho}\right) \beta_{\ell}=\Lambda_{\rho}$ is a semaphore code.

We can now prove that not all right congruences are special, even for $|A|=2$ :

Example 7.12 Let $A=\{a, b\}$ and let $\rho$ be the equivalence relation on $A^{3}$ defined by the following partition:

$$
\left\{a^{3}, a b a, b a^{2}\right\} \cup\left\{b a b, a^{2} b\right\} \cup\left\{a b^{2}\right\} \cup\left\{b^{2} a\right\} \cup\left\{b^{3}\right\} .
$$

Then $\rho \in \mathrm{RC}\left(A^{3}\right) \backslash \operatorname{SRC}\left(A^{3}\right)$.

Indeed, it is routine to check that $\rho \in \operatorname{RC}\left(A^{3}\right)$. Since $\operatorname{lcs}\left(a^{3} \rho\right)=a$ and $\operatorname{lcs}\left(\left(b^{2} a\right) \rho\right)=b^{2} a$, then $\Lambda_{\rho}$ is not a suffix code and so $\rho \notin \operatorname{SRC}\left(A^{3}\right)$ by Proposition 7.10.

Let $\rho \in \mathrm{RC}\left(A^{k}\right)$ and let

$$
\begin{aligned}
& \underline{\rho}=\vee\left\{\tau \in \operatorname{SRC}\left(A^{k}\right) \mid \tau \subseteq \rho\right\}, \\
& \bar{\rho}=\wedge\left\{\tau \in \operatorname{SRC}\left(A^{k}\right) \mid \tau \supseteq \rho\right\} .
\end{aligned}
$$

By Proposition 7.6(ii), we have $\underline{\rho}, \bar{\rho} \in \operatorname{SRC}\left(A^{*}\right)$.

Proposition 7.13 Let $|A|>1$ and $\rho \in \operatorname{RC}\left(A^{k}\right)$. Then:

(i) $\underline{\rho}=\tau_{\operatorname{Res}(\rho)}$; 
(ii) $\bar{\rho}=\tau_{A^{*} \Lambda_{\rho}}=\tau_{A^{*} \Lambda_{\rho}^{\prime}}$.

Proof. (i) By Lemma 7.8(ii), we have $\operatorname{Res}(\rho) \in \mathcal{I}_{k}(A)$. Now the claim follows from Proposition 7.9(ii).

(ii) Similarly, we have $A^{*} \Lambda_{\rho}=A^{*} \Lambda_{\rho}^{\prime} \in \mathcal{I}_{k}(A)$ by Lemma 7.7, and the claim follows from Proposition $7.9(\mathrm{i})$.

The next counterexample shows that the pair $(\underline{\rho}, \bar{\rho})$ does not univocally determine $\rho \in \operatorname{RC}\left(A^{k}\right)$ :

Example 7.14 Let $A=\{a, b\}$ and let $\rho, \rho^{\prime}$ be the equivalence relations on $A^{3}$ defined by the following partitions:

$$
\begin{aligned}
& \left\{a^{3}, a b a, b a^{2}\right\} \cup\left\{b a b, a^{2} b\right\} \cup\left\{a b^{2}\right\} \cup\left\{b^{2} a\right\} \cup\left\{b^{3}\right\}, \\
& \left\{a^{3}, b^{2} a, b a^{2}\right\} \cup\left\{b a b, a^{2} b\right\} \cup\left\{a b^{2}\right\} \cup\{a b a\} \cup\left\{b^{3}\right\} .
\end{aligned}
$$

Then $\rho, \rho^{\prime} \in \operatorname{RC}\left(A^{3}\right), \underline{\rho}=\underline{\rho^{\prime}}$ and $\bar{\rho}=\overline{\rho^{\prime}}$.

Indeed, we claimed in Example 7.12 that $\rho$ is a right congruence, and the verification for $\rho^{\prime}$ is also straightforward.

It is easy to see that

$$
\operatorname{Res}(\rho)=A^{*} A^{3} \cup\left\{a^{2}, a b\right\}=\operatorname{Res}\left(\rho^{\prime}\right),
$$

hence $\underline{\rho}=\underline{\rho^{\prime}}$ by Proposition 7.13(i).

Since

$$
\Lambda_{\rho}=\left\{a, a b, a b^{2}, b^{2} a, b^{3}\right\}
$$

and

$$
\Lambda_{\rho^{\prime}}=\left\{a, a b, a b^{2}, a b a, b^{3}\right\}
$$

we obtain

$$
A^{*} \Lambda_{\rho}=A^{+} \backslash\left\{b, b^{2}\right\}=A^{*} \Lambda_{\rho^{\prime}}
$$

and Proposition 7.13(ii) yields $\bar{\rho}=\overline{\rho^{\prime}}$.

This same example shows also that $\bar{\rho}$ does not necessarily equal or cover $\underline{\rho}$ in $\operatorname{SRC}\left(A^{k}\right)$. Indeed, in this case we have

$$
\operatorname{Res}(\rho)=A^{*} A^{3} \cup\left\{a^{2}, a b\right\} \subset I \subset A^{+} \backslash\left\{b, b^{2}\right\}=A^{*} \Lambda_{\rho}
$$

for $I=A^{*} A^{3} \cup\left\{a^{2}, a b, b a\right\} \in \mathcal{I}_{k}(A)$. By Lemma 7.4, we get

$$
\underline{\rho} \subset \tau_{I} \subset \bar{\rho} .
$$

\section{Random walks on semaphore codes}

As we have seen in Proposition 7.13, semaphore codes approximate right congruences from above and below in the lattice structure. In this section, we will define random walks (or more specifically Markov chains) on semaphore codes. The property that makes this possible is that for a semaphore code $S$ associated to the alphabet $A$

$$
S A \subseteq A^{*} S,
$$

see Lemma 4.1. Namely, (8.1) implies a right action of $A$ on $S$ : for $a \in A$ and $s \in S$, the action $s . a$ is $t$, if $s a=w t$ with $w \in A^{*}$ and $t \in S$ under (8.1). 
To turn the action $S \times A \rightarrow S$ into a random walk, we impose a Bernoulli distribution on $A^{*}$, see [6, Section 1.11]. More precisely, we associate a probability $0 \leq \pi(a) \leq 1$ to each letter $a \in A$ such that $\sum_{a \in A} \pi(a)=1$. The state space of the random walk is $S$. Given $s \in S$, with probability $\pi(a)$ we transition to state $s . a$ in one step. This gives rise to the transition matrix $\mathcal{T}$ with entry in row $s$ and column $s^{\prime}$

$$
\mathcal{T}_{s, s^{\prime}}=\sum_{\substack{a \\ \text { with } s^{\prime}=s . a}} \pi(a) .
$$

Since $\sum_{a} \pi(a)=1$, it follows that the row sums of $\mathcal{T}$ are equal to one, so that $\mathcal{T}$ is a row stochastic matrix. Taking $\ell$ steps in the random walk is described by the $\ell$-th power of $\mathcal{T}$, that is, the probability of going from $s$ to $s^{\prime}$ in $\ell$ steps is the $\left(s, s^{\prime}\right)$-entry $\left(\mathcal{T}^{\ell}\right)_{s, s^{\prime}}$ in $\mathcal{T}^{\ell}$. Under the Bernoulli distribution, the probability $\pi\left(a_{1} \cdots a_{\ell}\right)$ of a word of length $\ell$ is given by the multiplicative formula $\pi\left(a_{1} \cdots a_{\ell}\right)=$ $\prod_{i=1}^{\ell} \pi\left(a_{i}\right)$.

A suffix code $X$ on $A^{*}$ is maximal if it is not properly contained in any other suffix code on $A^{*}$, that is, if $X \subseteq Y \subseteq A^{*}$ and $Y$ is a suffix code, then $Y=X$. Furthermore, $X$ is called thin if there exists an elements $w \in A^{*}$ such that $A^{*} w A^{*} \cap X=\emptyset$. By [6, Proposition 3.3.10], for a thin maximal suffix code $X$ we have $\pi(X)=\sum_{x \in X} \pi(x)=1$ for all positive Bernoulli distributions $\pi$ on $X$. A Bernoulli distribution on $X$ is positive if $\pi(x)>0$ for all $x \in X$. As shown in [6, Proposition 3.5.1], semaphore codes $S$ are thin maximal suffix codes, so that

$$
\pi(S)=\sum_{s \in S} \pi(s)=1
$$

Hence any positive Bernoulli distribution on semaphore codes yields a probability distribution.

A stationary distribution $I=\left(I_{s}\right)_{s \in S}$ is a vector such that $\sum_{s \in S} I_{s}=1$ and $I \mathcal{T}=I$, that is, it is a left eigenvector of the transition matrix with eigenvalue one. In the finite state case, by the Perron-Frobenius Theorem, the stationary distribution exists. It is unique if the random walk is irreducible. See [13] for more details. In our case, we prove next that a stationary distribution exists and give its explicit form.

Theorem 8.1 The stationary distribution of the random walk associated to the semaphore code $S$ is given by

$$
I=(\pi(s))_{s \in S} .
$$

Proof. Taking the $s^{\prime}$-th component of $I \mathcal{T}=I$ reads

$$
\sum_{s \in S} \sum_{\substack{a \in A \\ s^{\prime}=s . a}} \pi(a) \pi(s)=\pi\left(s^{\prime}\right) .
$$

Recall that $s . a=s^{\prime}$ with $a \in A$ and $s, s^{\prime} \in S$ means that $s a=w s^{\prime}$ for some $w \in A^{*}$. In particular, this can only hold if $a$ is the last letter of $s^{\prime}$ and hence fixed by $s^{\prime}$.

Claim: The set $S^{\prime}=\left\{w \mid s a=w s^{\prime}, s \in S\right\}$ for fixed $s^{\prime} \in S$ with $a \in A$ the last letter of $s^{\prime}$, is a thin maximal suffix code.

Indeed, if the claim is true, we have $\sum_{w \in S^{\prime}} \pi(w)=1$ by [6, Proposition 3.3.10]. Using that $\pi(a) \pi(s)=\pi(w) \pi\left(s^{\prime}\right)$ we can hence rewrite (8.3)

$$
\sum_{s \in S} \sum_{\substack{a \in A \\ s^{\prime}=s . a}} \pi(a) \pi(s)=\pi\left(s^{\prime}\right) \sum_{w \in S^{\prime}} \pi(w)=\pi\left(s^{\prime}\right)
$$


as desired. It remains to prove the claim.

First assume that $S^{\prime}$ is not a suffix code. Then there must be two elements $w, w^{\prime} \in S^{\prime}$ that are comparable in suffix order. But then $w s^{\prime}$ and $w^{\prime} s^{\prime}$ are comparable in suffix order, contradicting the fact that $S$ is a suffix code (since after removing the last letter $a$ the result must be in $S$ ). Next assume that $S^{\prime}$ is not maximal. This means there exists $y \in A^{*}$ such that $S^{\prime} \subsetneq S^{\prime} \cup\{y\}$ is a suffix code. But then $S \cup\left\{y \widetilde{s}^{\prime}\right\}$ is a suffix code, where $\widetilde{s}^{\prime}$ is obtained from $s^{\prime}$ by removing the last letter $a$, contradicting the maximality of $S$ (recall that all semaphore codes are maximal by [6, Proposition 3.5.1]). Finally assume that $S^{\prime}$ is not thin. That means that there exists $w \in A^{*}$ such that $A^{*} w A^{*} \cap S^{\prime} \neq \emptyset$. In particular $u w v \in S^{\prime}$ for some $u, v \in A^{*}$. Since by construction $S^{\prime} \widetilde{s}^{\prime} \subseteq S$, this would imply $u w v \widetilde{s}^{\prime} \in S$, contradicting the fact that $S$ is thin.

Given $A=\left\{a_{1}, \ldots, a_{g}\right\}$ and a right congruence $\rho \in \mathrm{RC}\left(A^{k}\right)$, we are interested in the probability for nonempty words of length $\ell \leq k$ to be resets on $A^{k} / \rho$. Since $\operatorname{Res}(\rho)=\operatorname{Res}(\rho)$ by Propositions 7.10 and 7.13, we can restrict ourselves to determine the probabilities for resets of words of given length for $\underline{\rho} \in \operatorname{SRC}\left(A^{k}\right)$, or equivalently for semaphore codes $\Lambda_{\underline{\rho}}$ by Corollary 7.11.

Theorem 8.2 Let $\rho \in \mathrm{RC}\left(A^{k}\right)$. Then the probability that a word of length $1 \leq \ell \leq k$ is a reset on $A^{k} / \rho$ is given by

$$
P(\ell)=\sum_{\substack{s \in \Lambda_{\rho} \\ \ell(s) \leq \ell}} \prod_{a \in s} \pi(a),
$$

where $a \in s$ in the product runs over every letter in $s$ and $\ell(s)$ is the length of the word (or suffix) $s$.

Proof. As mentioned above, $\operatorname{Res}(\rho)=\operatorname{Res}(\underline{\rho})$ by Propositions 7.10 and 7.13 and in addition $\Lambda_{\underline{\rho}}$ is a semaphore code. Define $\operatorname{Res}(\ell)=\left\{w \in A^{+} \mid \ell(w)=\ell\right.$ and $w$ is a reset on $\left.A^{k} / \rho\right\}=\operatorname{Res}(\rho) \cap A^{\bar{\ell}}$. We claim that

$$
\operatorname{Res}(\ell)=\left\{w \in A^{+} \mid \ell(w)=\ell \text { and } w \text { has a suffix in } \Lambda_{\underline{\rho}}\right\} .
$$

Since $\Lambda_{\underline{\rho}}$ is a suffix code, each word has precisely one suffix in $\Lambda_{\underline{\rho}}$. Hence the claim immediately yields the formula for $P(\ell)$ using that a letter $a \in s$ for $s \in \Lambda_{\rho}$ occurs with probability $\pi(a)$.

We prove the claim by induction on $\ell$. By Proposition 7.10 (vii) we have that $\Lambda_{\underline{\rho}} \subseteq \operatorname{Res}(\underline{\rho})=$ $\operatorname{Res}(\rho)$. Certainly, for $\ell=1$ the only words that are resets are the words/suffixes of length 1 in $\Lambda_{\rho}$. Now assume that the claim holds for all words of length less than $\ell$. Since $\Lambda_{\underline{\rho}} \subseteq \operatorname{Res}(\rho)$, we deduce that

$$
\left\{w \in A^{+} \mid \ell(w)=\ell \text { and } w \text { has a suffix in } \Lambda_{\underline{\rho}}\right\} \subseteq \operatorname{Res}(\ell) .
$$

To prove the reverse inclusion let $v=a_{i_{\ell}} \ldots a_{i_{1}} \in \operatorname{Res}(\ell)$. If $v \in \Lambda_{\underline{\rho}}$, we are done. If $a_{i_{\ell-1}} \cdots a_{i_{1}} \in$ $\operatorname{Res}(\ell-1)$, then by induction $v$ has a suffix in $\Lambda_{\underline{\rho}}$. Hence assume that $a_{i_{\ell-1}} \ldots a_{i_{1}} \notin \operatorname{Res}(\ell-1)$ and $v \notin \Lambda_{\rho}$. This requires that $a_{i_{\ell}} \ldots a_{i_{2}}$ is a reset, so that again by induction $a_{i_{\ell}} \ldots a_{i_{2}}$ has a suffix $s$ in $\Lambda_{\underline{\rho}}$. Since $\Lambda_{\underline{\rho}}$ is a semaphore code and hence $\Lambda_{\underline{\rho}} A \subseteq A^{*} \Lambda_{\underline{\rho}}$, we have that if $s \in \Lambda_{\underline{\rho}}$, then $s a_{i_{1}} \in A^{*} \Lambda_{\underline{\rho}}$. In all cases $\bar{v}$ has a suffix in $\Lambda_{\underline{\rho}}$. This concludes the proof of the claim.

Example 8.3 Take the special right congruence $\rho$ given by congruency classes $\{a a a, b a a, a b a, b b a\}$, $\{a a b, b a b\},\{a b b\},\{b b b\}$ with corresponding semaphore code $\Lambda_{\rho}=\{a, a b, a b b, b b b\}$. The probability to have a reset for words of length $\ell$ is

$$
\begin{aligned}
& P(1)=\pi(a) \\
& P(2)=\pi(a)+\pi(a) \pi(b) \\
& P(3)=\pi(a)+\pi(a) \pi(b)+\pi(a) \pi(b)^{2}+\pi(b)^{3}=\pi(a)+\pi(a) \pi(b)+\pi(b)^{2}=\pi(a)+\pi(b)=1,
\end{aligned}
$$


where for $P(3)$ we have used repeatedly that $\pi(a)+\pi(b)=1$.

Example 8.4 Take the semaphore code

$$
\{a a, a a b, a b a, a b b a, b a b b, a a b b, b b a b, a b a b, b b b a, a a b b, b a b b b, a b b b b, b b b b b\}
$$

which corresponds to a special right congruence, which is easy to check by Proposition 7.10. Then we have

$$
\begin{aligned}
P(1) & =0 \\
P(2) & =\pi(a)^{2} \\
P(3) & =\pi(a)^{2}+2 \pi(a)^{2} \pi(b) \\
P(4) & =\pi(a)^{2}+2 \pi(a)^{2} \pi(b)+3 \pi(a)^{2} \pi(b)^{2}+3 \pi(a) \pi(b)^{3}=\pi(a)^{2}+2 \pi(a)^{2} \pi(b)+3 \pi(a) \pi(b)^{2} \\
& =\pi(a)^{2}+2 \pi(a) \pi(b)+\pi(a) \pi(b)^{2}=\pi(a)+\pi(a) \pi(b)+\pi(a) \pi(b)^{2} \\
P(5) & =\pi(a)+\pi(a) \pi(b)+\pi(a) \pi(b)^{2}+\pi(a)^{2} \pi(b)^{3}+2 \pi(a) \pi(b)^{4}+\pi(b)^{5} \\
& =\pi(a)+\pi(a) \pi(b)+\pi(a) \pi(b)^{2}+\pi(a) \pi(b)^{3}+\pi(b)^{4} \\
& =\pi(a)+\pi(a) \pi(b)+\pi(a) \pi(b)^{2}+\pi(b)^{3}=\pi(a)+\pi(a) \pi(b)+\pi(b)^{2} \\
& =\pi(a)+\pi(b)=1
\end{aligned}
$$

where again we repeatedly used that $\pi(a)+\pi(b)=1$.

The probability $P(\ell)$ to reach a reset in $\ell$ steps is related to the hitting time (see [13, Chapter 10]). Namely, given a Markov chain with state space $S$, the hitting time $t_{R}$ of a subset $R \subseteq S$ is the first time one of the nodes in $R$ is visited by the chain. We are interested in the hitting time $t_{\operatorname{Res}(\rho)}$ for $\rho \in \mathrm{RC}\left(A^{k}\right)$. Set

$$
p(\ell)=P(\ell)-P(\ell-1)=\sum_{\substack{s \in \Lambda_{\underline{\rho}} \\ \ell(s)=\ell}} \prod_{a \in s} \pi(a) .
$$

Then

$$
t_{\operatorname{Res}(\rho)}=\sum_{\ell=1}^{k} \ell p(\ell)
$$

Note that by Definition 2.2, we also have a right action of $A$ on right congruences $\rho \in \operatorname{RC}\left(A^{k}\right)$, namely $\rho \times A \rightarrow \rho$. Hence, as for semaphore codes, we can define a random walk on $\rho$ by assigning a probability $\pi(a)$ for each $a \in A$. Recall that by its definition in (7.7), $\underline{\rho}$ is a refinement of $\rho$. Let us relate these various random walks. A step $s . a=t$ for $s, t \in \Lambda_{\underline{\rho}}$ and $\bar{a} \in A$ in the random walk on the semaphore code $\Lambda_{\underline{\rho}}$ is in one-to-one correspondence to a step $c_{s} . a=c_{t}$ in the random walk on $\underline{\rho} \in \operatorname{SRC}\left(A^{*}\right)$, where $\bar{c}_{s}, c_{t} \in \underline{\rho}$ are the unique congruences such that $\operatorname{lcs}\left(c_{s}\right)=s, \operatorname{lcs}\left(c_{t}\right)=t$, respectively. Since $\underline{\rho}$ is a refinement of $\rho$, a step $c_{s} \cdot a=c_{t}$ on $\underline{\rho}$ implies a step $c . a=d$ on $\rho$ whenever $c_{s} \subseteq c$ and $c_{t} \subseteq d$. In particular, the transition matrix $\mathcal{T}$ for the random walk on the semaphore code $\Lambda_{\underline{\rho}}$ satisfies for a fixed $d \in \rho$

$$
\sum_{\substack{t \in \Lambda_{\rho} \\ c_{t} \subseteq \bar{d}}} \mathcal{T}_{s, t}=\sum_{\substack{t \in \Lambda_{\rho} \\ c_{t} \subseteq \bar{d}}} \mathcal{T}_{s^{\prime}, t} \quad \text { for all } s, s^{\prime} \in \Lambda_{\underline{\rho}} \text { such that } c_{s^{\prime}} \rho c_{s}
$$


This relation is precisely the condition for a Markov chain to be lumpable. Lumpability was first introduced by Kemeny and Snell [12], see also [13, Section 2.3.1]. This means that the transition matrix $\mathcal{T}^{\rho}$ on $\rho$ indexed by right congruence classes $c, d \in \rho$ can be expressed in terms of $\mathcal{T}$ as follows

$$
\mathcal{T}_{c, d}^{\rho}=\sum_{\substack{t \in \Lambda_{\rho} \\ c_{t} \subseteq \bar{d}}} \mathcal{T}_{s, t} \quad \text { for any } s \in \Lambda_{\underline{\rho}} \text { such that } c_{s} \subseteq c .
$$

The theory of lumpability (or projection) then gives us the stationary distribution $I^{\rho}$ for $\mathcal{T}^{\rho}$.

Proposition 8.5 Let $I^{\rho}=\left(I_{c}^{\rho}\right)_{c \in \rho}$ be the stationary distribution for $\mathcal{T}^{\rho}$. Then

$$
I_{c}^{\rho}=\sum_{\substack{s \in \Lambda_{\underline{\rho}} \\ c_{s} \subseteq c}} \pi(s) .
$$

Proof. By lumpability, we have

$$
I_{c}^{\rho}=\sum_{\substack{s \in \Lambda_{\underline{\rho}} \\ c_{s} \subseteq \underline{c}}} I_{s},
$$

where $I=\left(I_{s}\right)_{s \in \Lambda_{\underline{\rho}}}$ is the stationary distribution of $\mathcal{T}$. By Theorem 8.1 we have $I_{s}=\pi(s)$.

Remark 8.6 We could have derived an expression for $I^{\rho}$ also directly from the stationary distribution of the delay de Bruijn random walk by lumping given as

$$
I_{c}^{\rho}=\sum_{x \in c} \pi(x)
$$

\section{References}

[1] A. Arnold, A syntactic congruence for rational $\omega$-languages, Theoret. Comput. Sci. 39 (1985), no. 2-3, 333-335. 2

[2] A. Ayyer, J. Bouttier, S. Corteel, and F. Nunzi, Multivariate juggling probabilities, Electron. J. Probab. 20 (2015), no. 5, 1-29. 1

[3] A. Ayyer, S. Klee, and A. Schilling, Combinatorial Markov chains on linear extensions, J. Algebraic Combinatorics 39(4) (2014) 853-881. 10

[4] A. Ayyer, A. Schilling, B. Steinberg, and N. M. Thiéry, Markov chains, $\mathscr{R}$-trivial monoids and representation theory, Internat. J. of Algebra Comput. 25 (2015) 69-231. 10

[5] A. Ayyer and V. Strehl, Stationary distribution and eigenvalues for a de Bruijn process, In Ilias S. Kotsireas and Eugene V. Zima, editors, Advances in Combinatorics, pages 101-120. Springer Berlin Heidelberg, 2013. 1, 2

[6] J. Berstel, D. Perrin and C. Reutenauer, Codes and automata, Encyclopedia of Mathematics and its Applications 129, Cambridge University Press, Cambridge, 2010. 4, 5, 16, 17, 30, 31

[7] K. S. Brown, Semigroups, rings, and Markov chains, J. Theoret. Probab. 13(3) (2000) 871-938. 10 
[8] K. S. Brown and P. Diaconis, Random walks and hyperplane arrangements, Ann. Probab. 26(4) (1998) 1813-1854. 10

[9] N. G. de Bruijn, A combinatorial problem, Nederl. Akad. Wetensch., Proc. 49 (1946) 758-764. 1

[10] I. J. Good, Normal recurring decimals, J. London Math. Soc. 21 (1946) 167-169. 1

[11] K. Krohn and J. Rhodes, Algebraic theory of machines. I. Prime decomposition theorem for finite semigroups and machines, Trans. Amer. Math. Soc. 116 (1965) 450-464. 9, 10

[12] J. G. Kemeny and J. L. Snell, Finite Markov chains, Reprinting of the 1960 original. Undergraduate Texts in Mathematics. Springer-Verlag, New York-Heidelberg, 1976. ix+210 pp. 3, 33

[13] D. A. Levin, Y. Peres, and E. L. Wilmer, Markov chains and mixing times, American Mathematical Society, Providence, RI, 2009. With a chapter by James G. Propp and David B. Wilson. $3,30,32,33$

[14] O. Maler and L. Staiger, On syntactic congruences for $\omega$-languages, STACS 93 (Würzburg, 1993), 586-594, Lecture Notes in Comput. Sci., 665, Springer, Berlin, 1993. 2

[15] O. Maler and L. Staiger, On syntactic congruences for $\omega$-languages, Theoret. Comput. Sci. 183 (1997), no. 1, 93-112. 2

[16] A. Ralston, de Bruijn sequences - a model example of the interaction of discrete mathematics and computer science, Math. Mag. 55 (1982), no. 3, 131-143. 1

[17] J. Rhodes, Monoids acting on trees: elliptic and wreath products and the holonomy theorem for arbitrary monoids with applications to infinite groups, Internat. J. Algebra Comput. 1 (1991), no. $2,253-279.6$

[18] J. Rhodes, Applications of automata theory and algebra. Via the mathematical theory of complexity to biology, physics, psychology, philosophy, and games, With an editorial preface by Chrystopher L. Nehaniv and a foreword by Morris W. Hirsch. World Scientific Publishing Co. Pte. Ltd., Hackensack, NJ, 2010. xviii+274 pp. 2, 9, 14

[19] J. Rhodes and P. V. Silva, Turing machines and bimachines, Theoret. Comput. Sci. 400 (2008), no. $1-3,182-224.2$

[20] J. Rhodes and P. V. Silva, Further results on monoids acting on trees, Internat. J. Algebra Comput. 22 (2012), no. 4, 1250034, 69 pp. 6, 8

[21] J. Rhodes and B. Steinberg, The q-theory of finite semigroups, Springer Monographs in Mathematics, Springer, 2009. 9, 10, 11, 12, 13, 14

[22] J. Rhodes, A. Schilling and P. V. Silva, The semaphore codes attached to a Turing machine via resets and their various limits, preprint 2016. 5

[23] P. Stiffler, Jr., Extension of the fundamental theorem of finite semigroups, Advances in Mathematics, 11 (1973), no. 2, 159-209. 14 